\title{
Kernos
}

Revue internationale et pluridisciplinaire de religion grecque antique

29 | 2016

Varia

\section{Jeux de l'amour et du hasard en Grèce ancienne}

\section{Véronique Dasen}

\section{(2) OpenEdition \\ Journals}

\section{Édition électronique}

URL : http://journals.openedition.org/kernos/2390

DOI : 10.4000/kernos.2390

ISSN : 2034-7871

\section{Éditeur}

Centre international d'étude de la religion grecque antique

\section{Édition imprimée}

Date de publication : 1 octobre 2016

Pagination : 73-100

ISSN : 0776-3824

\section{Référence électronique}

Véronique Dasen, « Jeux de l'amour et du hasard en Grèce ancienne », Kernos [En ligne], 29 | 2016, mis en ligne le 01 octobre 2019, consulté le 10 décembre 2020. URL : http://journals.openedition.org/ kernos/2390 ; DOI : https://doi.org/10.4000/kernos.2390

Ce document a été généré automatiquement le 10 décembre 2020.

Kernos 


\title{
Jeux de l'amour et du hasard en Grèce ancienne
}

\author{
Véronique Dasen
}

Une première version de cet article a été présentée dans le cadre du séminaire "Anthropologie et image dans les mondes anciens : jeu et genre » donné en hiver 2015 à l'EHESS avec François Lissarrague et je remercie les participants pour leurs échanges, en particulier Françoise FrontisiDucroux, Roberte Hamayon, Philippe Moreau et Marie-Christine Villanueva-Puig.

1 Sur les vases attiques et italiotes, les peintres de l'époque classique ont représenté plusieurs jeux dont les acteurs privilégiés sont des jeunes gens en âge de se marier. Cet univers ludique transpose le prélude aux relations amoureuses et les préparatifs du mariage dans un espace virtuel. La référence au double sens du verbe $\pi \alpha i \zeta \omega$, qui signifie à la fois « jouer » et "badiner », est parfois explicite, comme dans une variante

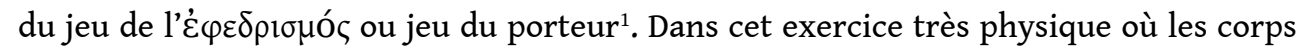
se touchent, quand un joueur porte l'autre sur son dos, les partenaires sont en principe toujours de même sexe pour des raisons de pudeur. Seules des figures masculines surnaturelles, comme Éros, se mêlent parfois au monde féminin. Sur une des faces d'un skyphos apulien (Fig. 1 ; vers 375-350 av. J.-C.) ${ }^{2}$, le dieu est monté sur le dos d'une jeune femme, le genou gauche posé dans la boucle que forment les bras de la belle tout en lui masquant les yeux de ses mains. La femme est richement parée comme une numphê désirable, prête à se marier, vêtue d'un fin chiton sans manche, ornée d'un collier et de bracelets. Elle s'avance courbée sous le poids d'Éros et progresse à tâtons en direction $\mathrm{du}$ but à atteindre, une sorte d'empilement de cailloux peints en rehaut blanc. Le jeu fait référence au mariage pensé comme la domestication d'une parthenos souvent

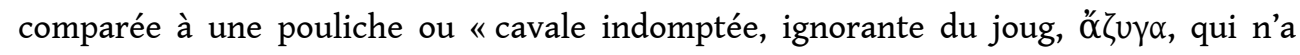
connu ni le mâle, ni l'amour $»^{3}$. Le groupe symbolise à la fois l'aveuglement de l'amour, dont Éros est tenu pour responsable, l'incertitude qu'il provoque, et la soumission aux forces contraignantes qu'exercent Aphrodite et Éros. Le mouvement de marche met en image le processus de transition en train de s'opérer. Au-dessus de la cible, une bandelette frangée est suspendue. Elle évoque la ceinture qui symbolise la réussite au féminin de l'agôn amoureux en tant qu'élément de la parure de la mariée; elle 
accompagne le changement du statut de jeune fille à celui d'épouse, puis, dénouée pour l'accouchement, le passage au statut de gunê, l'épouse accomplie par la naissance du premier enfant ${ }^{4}$. À sa manière, elle représente l'équivalent du bandeau qui couronne l'athlète vainqueur. Sur l'autre face, la scène résume la beauté du couple idéal. L'homme est debout, nu, un himation négligemment drapé sur ses épaules, la tête ceinte d'un bandeau, face à une jeune fille assise; il tient un strigile, attribut d'un corps athlétique. La femme, ornée de boucles d'oreilles, collier et bracelets, vêtue d'une fine étoffe qui épouse ses formes, porte sur le dos de sa main un oiseau, peut-être une colombe, emblème de sa grâce et de la présence d'Aphrodite 5 . Dans le champ de l'image, au-dessus de la tête de la jeune fille, un motif circulaire en forme de rosette pourrait faire allusion au jeu de balle. Un skyphos similaire de l'atelier du Peintre de l'Ilioupersis confirme cette interprétation nuptiale. Sur une face, Éros verse l'eau du bain prénuptial sur la jeune fille nue accroupie devant un bassin, sur l'autre figure un couple dont l'homme est debout et la femme assise, un oiseau sur la main ${ }^{6}$.

2 L'iconographie d'autres jeux d'adresse construit un espace où les jeunes filles ne sont pas perçues comme soumises à leur destin. Le badinage se traduit visuellement par un agôn érotique qui leur donne une part active et se transforme parfois en procédure divinatoire, voire magique, sous le regard d'Éros et d'Aphrodite ${ }^{7}$. Tout jeu d'adresse constitue en effet une sorte de consultation oraculaire dans la mesure où son issue dépend de la bienveillance des dieux qui régissent le hasard. Dans l'iconographie vasculaire, leur représentation peut traduire une façon culturelle, largement partagée, de penser le destin en contexte amoureux. Nous examinerons le double sens métaphorique de trois jeux : le jeu de balles, de toupie et de morra ou «tirage au sort par les doigts ».

\section{Jeux de balles, pommes, pelotes...}

3 Le motif de la femme jonglant avec plusieurs objets ronds, des pommes, balles ou pelotes de laine, apparaît dans le deuxième tiers du v siècle av. J.-C. sur la peinture de vases attiques ${ }^{8}$. Ce type de scène succède à celles de récoltes de fruits dans un verger, une activité collective, accomplie exclusivement par des femmes dans l'imagerie de l'époque archaïque. Quelques représentations de la fin du vie siècle av. J.-C. témoignent des étapes du glissement du motif. Les femmes sont réunies, debout ou assises, dans un espace qui semble être cette fois dévolu au travail domestique. Le grand panier à fond arrondi, qui contenait les fruits, a désormais la forme allongée du kalathos, le panier à laine; le pommier a disparu, mais la présence d'un rameau végétal pourrait être une réminiscence du motif du verger ${ }^{9}$.

Vers 480 av. J.-C., l'intérêt des imagiers se concentre sur l'espace du gynécée, défini par la présence d'un klismos ou chaise à dossier, et du kalathos, le panier à laine qui fait référence aux vertus du travail domestique. Sur le lécythe attribué au Peintre de Karlsruhe, la jeune femme semble se distraire en jonglant avec trois objets ronds que des auteurs modernes identifient à des pelotes de laine à cause du kalathos posé à côté d'elle (Fig. 2 ; vers $480-470$ av. J.-C.) ${ }^{10}$. Le panier est cependant parfois absent. Sur la pyxis du Peintre de Pistoxenos (vers 480-470 av. J.-C.) ${ }^{11}$, six femmes sont assises avec des accessoires qui indiquent leur statut d'épouses ou de futures épouses de citoyen. $\mathrm{Au}$ centre, deux femmes se font face. À gauche, l'une d'elles tient une couronne végétale, la tête inclinée avec pudeur, comme une numphê, l'autre jongle avec des objets sphériques 
indéterminés ${ }^{12}$; en l'absence de kalathos, s'agit-il de pelotes de laine, de balles ou de pommes ? Un couple similaire se retrouve sur une pyxis conservée à Oxford ${ }^{13}$ : sur une face, une jeune femme assise jongle face à une autre entièrement enveloppée dans son himation dont n'émerge qu'une main portant une fleur à son nez. Aucun kalathos n'est présent ; les paniers à laine ne sont figurés qu'au revers, associés à trois femmes assises filant la laine. L'apparence du kalathos entretient parfois l'ambiguïté de l'accessoire de jonglage. Sur le médaillon d'une coupe du Peintre de Munich (vers $440 \mathrm{av.J.-C.),} \mathrm{le}$ panier de la jongleuse ressemble par sa taille et sa forme arrondie à celui utilisé pour la cueillette des fruits ${ }^{14}$.

5 Le jonglage prend une dimension érotique dès qu'un jeune homme apparaît. Le jeu semble alors constituer une sorte de communication non verbale entre les jeunes gens. Sur une amphore de la collection Sambon à Milan (Fig. 3 ; vers 450 av. J.-C. $)^{15}$, une jeune fille, vêtue d'un fin chiton, les cheveux retenus par un sakkos, marche en jonglant près d'une chaise; elle semble se diriger vers l'autre face où un jeune homme imberbe debout la contemple, immobile, les bras enveloppés dans son himation, en serrant son bâton de citoyen. Sur la coupe du Peintre de Penthésilée, conservée à Boston (Fig. 4 ; vers $460 \mathrm{av}$. J.-C. $)^{16}$, le couple est réuni dans un intérieur défini à gauche par la présence d'un kalathos d'où dépasse une quenouille, et au centre par une bandelette suspendue dans le champ de l'image. Un dialogue est suggéré par la pose du garçon debout, penché vers la jeune fille assise qui manipule, sans jongler, deux balles ou fruits qu'elle semble lui présenter.

6 L'identification de l'accessoire est sans doute rendue volontairement impossible. Dans les sources littéraires, pomme, $\mu \tilde{\eta} \lambda o v$, et balle, $\sigma \varphi \alpha \tilde{p} \rho \alpha$, peuvent se substituer l'une à l'autre en contexte érotique. Dans de nombreux récits, elles sont toutes deux les vecteurs des contacts entre filles et garçons. Dans l'odyssée ${ }^{17}$, le jeu de balle auquel s'adonnent Nausicaa et ses compagnes crée l'occasion de la rencontre avec Ulysse ; Athéna cause la maladresse qui fait perdre la balle dans une cascade, suscitant les cris excités des jeunes filles qui réveillent le héros.

7 Le rapport étroit de la pomme, $\mu \tilde{\eta} \lambda$ ov ou $\mu \tilde{\alpha} \lambda$ ov, avec Aphrodite est bien connu ${ }^{18}$. Les pommes sont les fruits par excellence de deux vergers célèbres, le kêpos d'Aphrodite à Paphos et celui des Hespérides qui portent des fruits d'or pour le mariage de Zeus et Héra ${ }^{19}$. L'image de la pomme qui mûrit symbolise la jeune fille prête au mariage, et la cueillette renvoie aux préparatifs des noces ${ }^{20}$. Dans le roman Daphnis et Chloé, Daphnis cueille pour Chloé une pomme chargée de la double référence au choix de Pâris et aux pommes d'or du jardin des Hespérides :

«C'est le prix qu'Aphrodite a reçu pour sa beauté ; c'est celui que je te donne pour ta victoire. Nous sommes dans la même situation pour témoigner en votre faveur, puisque lui, Pâris, était berger et que moi je suis chevrier ». À ces mots, il la dépose sur les genoux de Chloé. Et comme il s'était approché, elle lui donne un baiser; aussi Daphnis ne regretta pas d'être monté à une telle hauteur, puisqu'il reçut un baiser valant mieux qu'une pomme d'or ${ }^{21}$.

Chez de nombreux auteurs, le champ sémantique de $\mu \tilde{\eta} \lambda$ ov ou $\mu \tilde{\alpha} \lambda$ ov, qui désigne de manière générique un fruit rond et charnu, pomme, coing ou grenade, s'étend au corps féminin érotisé ; l'image de la pomme devient métaphore des seins ainsi que des joues, voire du clitoris ${ }^{22}$. Le lancer de la pomme, mêloboleîn, est une expression proverbiale synonyme de séduction de la part d'hommes comme de femmes ${ }^{23}$. Chez Théocrite, Cléariste jette au chevrier des pommes en le sifflant ${ }^{24}$, tandis que dans les Nuées un jeune homme reçoit le conseil de «[...] ne pas faire irruption chez une danseuse » pour 
éviter de "recevoir une pomme (mêlon) lancée par une petite catin», ce qui lui fera perdre sa bonne réputation ${ }^{25}$. Chez les poètes de l'Anthologie palatine, la pomme est une invite à la réciprocité du sentiment amoureux :

Je suis une pomme, mêlon; celui qui me jette, c'est quelqu'un qui t'aime. Accède à ses désirs Xanthippê ; nous sommes, toi comme moi, destinés à nous flétrir ${ }^{26}$.

Dans un des tableaux fictifs décrits par Philostrate, Éros fait irruption dans le jeu. Les Érotes récoltent des pommes dans le verger d'Aphrodite et se servent des fruits comme de balles qui véhiculent leurs baisers. Leur jeu symbolise «l'amour naissant $»^{27}$. Chez Méléagre, la balle devient le cœur avec lequel joue Éros :

C'est un joueur de balle, l'Amour que je nourris ; il te lance, Héliodôra, le cœur qui bondit en mon sein. Allons, accepte-le comme partenaire ; mais si de ton côté tu ne me renvoies pas le désir, je ne tolérerai pas cet affront contraire aux usages de la palestre ${ }^{28}$.

10 Cette dimension érotique de la cueillette trouve une expression visuelle chez quelques peintres du cercle du Peintre de Meidias à la fin du v viècle av. J.-C. Sur le cratère en cloche conservé à Boston ${ }^{29}$, deux Érotes font tomber les fruits de l'arbre et les récoltent en présence de la déesse et d'un couple de jeunes gens anonymes.

11 La pomme peut aussi concentrer un pouvoir supérieur qui dépasse la simple invite. Les pommes d'or du kêpos d'Aphrodite exercent une force contraignante qui permet à Hippomène de remporter la course contre Atalante, la parthenos indomptable, et de conquérir son cœur. Chez Théocrite, elle perd la tête en découvrant les fruits précieux, et tombe sous l'emprise d'un envoûtement amoureux :

Hippomène, briguant la vierge, prit les pommes et accomplit la course ; Atalante, à

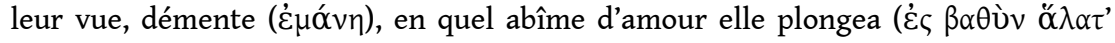

$\varepsilon \tilde{\varepsilon} \omega \tau \alpha)^{30}$.

La pomme est aussi un véritable instrument de magie érotique dans l'histoire des amours contrariées d'Acontios et Cydippe ${ }^{31}$. Dans le sanctuaire d'Artémis à Délos, Acontios lance à la jeune fille une pomme qui porte l'inscription : «Je jure par Artémis d'épouser Acontios». Cydippe fut ainsi capturée par une pomme, dit Ovide ${ }^{32}$. Cydippe attrape la pomme et prononce la phrase à voix haute, se liant ainsi involontairement par un serment prononcé dans un lieu sacré. Par la suite, elle tombe malade à chaque fois qu'un mariage va se conclure, jusqu'à ce que son père découvre l'existence du serment et accorde sa fille à Acontios ${ }^{33}$.

Les pommes entrent dans la préparation de philtres aphrodisiaques, comme celui conservé sur un papyrus grec d'époque augustéenne, à prononcer trois fois pour s'emparer de la femme désirée en suscitant la folie amoureuse, mania, le désir, erôs, l'amour, philia, l'affection, storgê. L'incantation se réfère au jeu: «Je lance des pommes ... Celle à qui je donne la pomme, à qui je jette la pomme, que je frappe », et invite à manipuler et consommer le fruit pour fixer le destin : «puisse-t-elle la plaçant dans sa main la manger $»^{34}$.

14 Les scènes de jonglage avec des balles, pelotes ou pommes ne sont donc pas à interpréter au premier degré. Elles ne mettent pas en scène un simple divertissement, mais une invitation à une relation amoureuse réciproque. Le discours allégorique des imagiers contraste sur un point important avec celui des textes. Dans les sources écrites, la tentative de séduction vient principalement d'un homme qui cherche à contraindre une femme. Dans l'iconographie, le jonglage est toujours pratiqué au féminin, révélant la part active des jeunes filles. Ce sont elles qui cherchent à susciter le 
désir des hommes, d'une façon pensée en termes agonistiques. L'imagier établit parfois une comparaison explicite avec l'entraînement des garçons. Sur un skyphos béotien (Fig. 5 ; dernier quart du $\mathrm{v}^{\mathrm{e}} \mathrm{s}$. av. J.-C. $)^{35}$, une jeune fille est assise sur un coffre qui évoque le trousseau de la mariée. Elle s'apprête à lancer une balle à des partenaires surnaturels, un petit Éros aux bras déjà tendus, juché sur les épaules d'un compagnon ${ }^{36}$ Le schéma de la scène est emprunté à celui d'un jeu de garçons dans la palestre. Sur un lécythe à figures noires attribué au Peintre d'Édimbourg (vers 500 av. J.-C.) ${ }^{37}$, l'entraîneur, plus âgé, est assis face à deux équipes de jeunes gens qui se chevauchent, comme les Érotes béotiens. Sur le skyphos béotien, l'agôn est transposé au féminin dans un espace nuptial imaginaire où la femme est dépeinte à la place du pédotribe, comme celle qui dirige l'exercice.

Seules ou à plusieurs, les jeunes filles ne jonglent-elles donc que pour se distraire ? L'exercice pourrait être une manière d'interroger et d'influencer leur avenir amoureux en agissant sur la séduction de l'être aimé. Le jonglage peut en effet s'apparenter à une procédure magique. Sur un fragment de lébès gamikos (Fig. 6 ; vers 430-420 av. J.-C.) ${ }^{38}$, un vase associé aux rites de mariage, une numphê jongle avec des objets de forme irrégulière qui pourraient être des pommes qui tournoient dans ses mains à la manière d'un charme érotique. Le mouvement rotatoire évoque celui de la iunx, l'instrument par excellence de la magie amoureuse, actionné pour attirer l'amant désiré39 également figuré dans les scènes de préparatifs de mariage. Sur une pyxis du Peintre d'Érétrie (vers 440-430 av. J.-C.) ${ }^{40}$, Pontomedeia, une Néréide, tient les lanières du disque qu'elle fait tournoyer pour assurer une union heureuse, liée par Peithô, la Persuasion ${ }^{41}$. Sur le médaillon d'une coupe conservée à Chicago (Fig. $7 ; 440-420$ av. J.-C. $)^{42}$, un Éros vient couronner la jongleuse assise sur un diphros, comme pour récompenser son adresse qui va de pair avec sa capacité de susciter le désir, et par là d'attirer un amant.

\section{La toupie ou le vertige de l'amour}

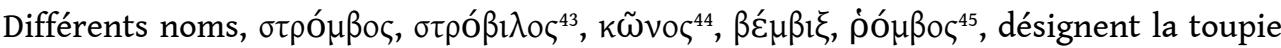
et le sabot, des objets de forme conique avec la capacité de tourner sur eux-mêmes en équilibre sur la pointe, mus par une force extérieure. La toupie est actionnée par la ficelle qui l'entoure, déroulée d'un coup sec, le sabot par les lanières d'un fouet, mastix, qui le relance chaque fois qu'il se ralentit en le faisant se déplacer rapidement dans l'espace ${ }^{46}$. La classe d'âge qui s'y adonne est à nouveau celle des jeunes gens qui offrent ce jouet à Hermès, le dieu à la démarche sinueuse comme la trajectoire du jouet ${ }^{47}$.

Les imagiers grecs semblent avoir utilisé l'objet comme un opérateur métaphorique correspondant aux différents champs de compétence d'un dieu associé à la paideia et à une forme particulière de mantique. Dans trois scènes, Hermès actionne lui-même le sabot. Sur le médaillon d'une coupe à figures rouges attribuée à Douris (vers 480 av. J.C. $)^{48}$, le dieu, vêtu d'une chlamys et d'un pétase, fait face à un jeune homme drapé dans son himation. Les deux personnages sont penchés sur le sabot posé sur le sol au centre de la scène. Hermès lève le bras droit d'un geste ample suggérant la force et la précision du coup de fouet qui va s'abattre. Il démontre sa maitrise des mouvements aléatoires du jouet, à l'image des "forces incontrôlées de l'adolescence " dont il est un des gardiens dans le gymnase ${ }^{49}$. L'excitation du garçon est traduite par sa main ouverte tendue vers l'objet avec une spontanéité juvénile ${ }^{50}$. L'espace circulaire du médaillon vient renforcer la suggestion de la rotation du sabot dont les rayures devaient s'animer 
en tournant ${ }^{51}$. Le contrôle du mouvement de l'objet renvoie à la nécessité pour les jeunes gens de faire l'apprentissage de la maîtrise de soi $^{52}$. Le vertige non contrôlé peut être mortel. Dans des sources plus tardives, le petit Dionysos en est victime quand il est abusé par les Titans qui lui présentent trois sortes de toupies, rhombos, kônos, strobilos, avec des balles, spherai, pommes, mêla, et osselets, astragaloi, avant de le tuer et de le démembrer ${ }^{53}$.

Le fouet qui actionne le jouet possède aussi un double sens qui l'associe aux procédés de la magie amoureuse. Il fait pirouetter l'objet comme le cœur en proie à l'émotion. La description la plus explicite se trouve dans la poésie latine. Pour dire son trouble, Tibulle évoque le mouvement d'un sabot: «Car je suis agité comme le sabot que fait tourner rapidement sur un sol uni et libre le fouet preste d'un enfant exercé à ce jeu " ${ }^{54}$. L'effet des charmes amoureux est aussi comparé à celui d'un fouet. Dans les incantations des Papyrus grecs magiques, la victime doit perdre tout contrôle sous le fouet de la puissance invoquée qui lui donne une impulsion contraire à sa volonté, à l'image d'un sabot ${ }^{55}$. Chez Théocrite, la magicienne use à la fois d'une iunx et d'un rhombos d'airain qui « tourne éperdument sous l'action d'Aphrodite » pour contraindre l'être convoité ${ }^{56}$. Une série de vases italiotes met en scène la puissance exercée sur les sentiments amoureux par le mouvement de l'objet. Sur une péliké apulienne conservée au Musée de Matera (Fig. $8 ; 390-380$ av. J.-C. $)^{57}$, Éros se substitue à Hermès. Le jeune dieu, debout entre deux jeunes femmes richement vêtues, s'apprête à frapper le sabot posé par terre. Une des femmes est assise et observe attentivement le mouvement de l'objet, l'autre, debout derrière Éros, tient une balle. Sur un cratère en cloche apulien conservé à Syracuse ${ }^{58}$, Hermès est également présent; la jeune fille ne joue pas à la toupie, elle est devenue une toupie qu'Éros fait tournoyer en lui tenant les mains. Audessus du groupe, une bandelette est suspendue, peut-être en référence à la ceinture du mariage.

Le tournoiement de la toupie ou du sabot peut aussi détenir une valeur divinatoire de type cléromantique ou clédonomantique, comme d'autres signes produits par le hasard présidés par Hermès ${ }^{59}$. Dans une épigramme attribuée à Callimaque ${ }^{60}$, le jeu dévoile l'avenir amoureux. Aux mouvements aléatoires de la toupie s'ajoute le klêdôn, une parole spontanée qui constitue un message divin. Le présage concerne le choix d'un mariage. Un jeune homme vient consulter Pittacos de Mytilène, un des Sept Sages, car il hésite entre deux unions, l'une avec une femme plus fortunée que lui, l'autre de statut égal au sien. Le sage lui conseille d'observer le jeu d'enfants et d'écouter leurs paroles :

C'étaient des enfants qui, tenant sous leurs coups de rapides toupies, bembikes, les faisaient tourner sur une grande place. « Voilà ! dit-il, suis leurs traces. » - Le jeune homme s'approcha ; et les enfants disaient : "Mène celle qui est à ta portée ». À ce mot entendu, l'étranger se garda de rechercher une lignée supérieure : la parole des enfants fut sa règle ${ }^{61}$.

Quelques vases attiques ajoutent une dimension agonistique au jeu de la toupie. Les jeunes filles sont cette fois seules. Sur un lécythe conservé à New York (Fig. 9; 440-430 av. J.-C.) ${ }^{62}$, deux femmes se penchent sur leurs toupies dont elles suivent les mouvements avec concentration, étroitement enveloppées dans leur himation pour ne pas gêner leurs mouvements. Comme dans tout agôn, l'entraînement se fait entre pairs, de force égale ${ }^{63}$. Seuls de petits détails différencient les joueuses, l'une a les cheveux retenus dans un sakkos, l'autre les cheveux libres. La compétition consisterait-elle à faire tourner sa toupie le plus longtemps possible, avec un résultat de valeur divinatoire? La scène se retrouve sur un lécythe à fond blanc du Peintre de Sabouroff 
(460-450 av. J.-C.) $)^{64}$. Deux jeunes femmes s'affrontent autour d'une ou deux toupies. Le sens de la compétition pourrait dépasser ici l'évocation nostalgique de la vie quotidienne de la disparue. De manière métaphorique, le jeu pourrait représenter la part de l'aléatoire dans la vie, en comparant la durée imprévisible du mouvement de la toupie à celle de l'existence.

\section{Un jeu virtuel ?}

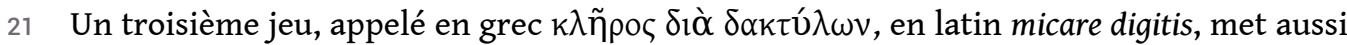
en scène l'imaginaire des jeunes gens, et plus particulièrement des filles, à la veille du mariage. Au premier abord, ses règles semblent être proches du jeu connu aujourd'hui sous le nom italien morra (en français «mourre »), un jeu d'adresse et de hasard joué à deux, sur le pourtour de la Méditerranée, simplement avec les mains mais dont la technique et les objectifs diffèrent ${ }^{65}$. La morra actuelle est un jeu de pari, souvent pour de l'argent, qui se pratique entre hommes d'âge adulte. Les joueurs sont debout ou assis, face à face. Chacun lève en même temps la main droite en indiquant un chiffre avec les doigts, et en criant à haute voix la somme totalisée avec son adversaire. Celui qui dit le premier le bon chiffre a gagné la manche. Le jeu se déroule de manière très rythmée et rapide pour éviter toute tricherie, sans laisser le temps de lever ou abaisser subrepticement un doigt pour avoir le bon résultat ${ }^{66}$. Le succès ne repose pas que sur la vivacité du joueur, mais sur son expérience, sa concentration et sa présence physique et sonore qui vise à intimider le concurrent.

Les principales sources écrites antiques qui décrivent cette activité sont d'époque romaine $^{67}$. L'expression qui la désigne, micare digitis, contient le verbe micare, " trembler ", "frémir ", " palpiter ", qui suggère sa dimension divinatoire ${ }^{68}$. Cicéron l'associe au tirage au sort par les osselets ou les dés :

Qu'est-ce qu'un sort? À peu près la même chose que le jeu de la «mourre », le lancer des osselets ou des dés. Jeux régis par le hasard et l'imprévu, non par la réflexion et le calcul. Toute cette technique a été inventée par tromperie, pour faire du profit, pour promouvoir la superstition ou induire en erreur ${ }^{69}$.

L'objectif de l'exercice est une prise de décision. Les dieux sont tenus responsables du geste qui détermine le succès. Des allusions suggèrent que le résultat pouvait être obtenu immédiatement, ou seulement après plusieurs tours. Dans un poème de Calpurnius Siculus, l'ordre de passage des bergers est déterminé après trois essais :

«Et maintenant, pour pouvoir mieux répartir l'alternance de vos chants, jetez chacun les mains par trois fois en les ouvrant brusquement ». Sans tarder, ils s'en remettent au jugement des doigts ${ }^{70}$.

Dans l'iconographie antique, comme dans l'exercice moderne du jeu, les joueurs sont généralement entourés de spectateurs attentifs qui surveillent le bon déroulement de la partie ${ }^{71}$. Le jeu attesté dans l'iconographie grecque dès le milieu du ve siècle av. J.-C., en Grèce continentale (Attique et Béotie) et en Grande Grèce (Apulie) ${ }^{72}$, présente des ressemblances avec celui décrit par les auteurs d'époque romaine, mais aussi de grandes différences. D'une part, les joueurs ne sont ni des enfants, ni des adultes, ni exclusivement des hommes, au contraire, la paire est d'ordinaire composée de jeunes gens en âge de se marier, de deux jeunes filles (Fig. 13), d'un garçon et d'une fille (Fig. 12) , et parfois de partenaires divins : la jeune fille joue avec Aphrodite (Fig. 14), Éros avec Niké ${ }^{73}$, Éros avec Aphrodite, ou deux Érotes ensemble (Fig. 10). Plus rarement, une 
ménade joue avec un satyre, ou des satyres ensemble (Fig.11). Le jeu se déroule d'ordinaire à l'extérieur, en contexte urbain, auprès d'une fontaine (Fig. 13), ou agreste, parmi des rochers (Fig. 10). Un accessoire absent des textes plus tardifs revient régulièrement: un bâton, relativement long et plus ou moins noueux, est tenu de la main gauche par les joueurs; il associe peut-être le tirage au sort aux pratiques du milieu des bergers.

Dans toutes les scènes, le mouvement des doigts semble indiquer un chiffre ${ }^{74}$, peut-être énoncé à voix haute, comme aujourd'hui. Sur l'hydrie de Goluchow, la femme de droite lève une main entièrement ouverte, tandis que celle de gauche lève deux doigts. Sur le cratère à volutes apulien du Peintre de Sisyphe (Fig. 10 ; vers 420 av. J.-C.) ${ }^{75}$, l'index levé de l'Éros de droite répond à la main levée de celui de gauche. Aucune information ne peut être tirée des expressions calmes sur l'oralité ou le rythme du jeu.

Une inscription indique parfois le résultat ${ }^{76}$. Sur un fragment de coupe de la collection H.A. Cahn, deux satyres vêtus d'un himation, comme des citoyens ordinaires, jouent à "bouge-doigts ", assis par terre dans un espace extérieur indiqué par un arbre (Fig.11; vers $450 \mathrm{av} . \mathrm{J} .-\mathrm{C}$. $)^{77}$. Le jeu se déroule en présence d'un spectateur, un troisième satyre, debout, appuyé sur son bâton de citoyen. Les partenaires tiennent chacun l'extrémité d'un bâton noueux. Le personnage de droite lève la main en indiquant un chiffre avec trois doigts levés, mais l'issue est encore indéterminée car les joueurs sont à égalité, comme le suggère la lettre epsilon désignant le chiffre 5 qui est peinte au-dessus de la tête de chacun. Les satyres sont réputés pour leur appétit sexuel. Sont-ils en train de tirer au sort la chasse à une partenaire érotique ? L'humour naît du décalage entre les vêtements d'adultes et la pose, assis par terre, qui infantilise les satyres. D'autres activités ludiques manifestent leur nature puérile, comme le jeu de l'ephedrismos ou du porteur qui n'est jamais pratiqué par des adultes ${ }^{78}$, ou ceux qu'ils pratiquent avec des enfants, en déviant du comportement attendu d'un père athénien ${ }^{79}$.

Certains détails pourraient suggérer le mode de déroulement du jeu, comme la position décalée des mains des partenaires sur le bâton (Fig. 10). Sur une lékané apulienne (Fig. 12 ; vers 380 av. J.-C. $)^{80}$, Éros couronne la jeune fille dont les doigts ont atteint l'extrémité du bâton, comme si les points se marquaient en reculant peu à peu la main, et que le premier arrivé au bout du bâton avait gagné la partie.

Dans presque toute la série, la scène se déroule dans un contexte prénuptial métaphorique, liant à nouveau le jeu de l'amour à celui du hasard. Le tirage au sort constitue le prélude d'une union amoureuse. Comme dans tout alea, le succès est dû à la bienveillance des dieux. Le type de siège des jeunes filles est ainsi sans rapport avec la pratique réelle du jeu. Sur la lékané apulienne (Fig. 12), comme sur l'ôon attique (Fig. 14), la joueuse est assise sur un grand coffre ouvragé qui fait allusion à la dot du mariage ${ }^{81}$. Sur la lékané apulienne (Fig. 12), deux rosettes placées à chaque extrémité de la scène pourraient faire allusion au jonglage avec des balles (ou s'agit-il d'une iunx ?). Sur une hydrie attique attribuée au Peintre du bain (Fig. 13 ; vers $440-420$ av. J.-C.) ${ }^{82}$, la scène est située aux abords d'une fontaine monumentale, rendue par une colonne, une marche et la bouche en forme de tête de lion, où deux jeune femmes séduisantes sont venues chercher de l'eau ${ }^{83}$. Au lieu d'exécuter leur tâche, elles sont assises sur les vases et s'adonnent au tirage au sort, comme pour traduire leur impatience de savoir laquelle se mariera la première. Plusieurs éléments font référence à la fête à venir, à commencer par la fontaine elle-même, procurant l'eau du bain nuptial, l'Éros qui apporte en volant un bandeau à la victorieuse et la femme qui lui tend une couronne végétale. 
Le contexte prénuptial est précisé sur un oôn du Cercle de Peintre d'Érétrie (Fig. 14 ; vers 420 av. J.-C.) ${ }^{84}$. Par sa forme ovoïde, ce petit vase pourrait avoir constitué un cadeau de mariage. La scène réunit beaucoup de personnages en mêlant le plan humain et divin. $\mathrm{Au}$ centre, une jeune femme, coiffée d'un diadème, ornée de boucles d'oreilles et de bracelets, joue avec Aphrodite, dont la grande taille signale le statut divin, accompagnée d'Éros, qui se blottit contre elle en lui adressant un regard complice. Derrière la déesse se tient un jeune homme debout, vêtu d'une chlamys, portant deux lances et coiffé d'un pétase, qui pourrait représenter le futur époux. La jeune femme est entourée de trois compagnes qui semblent suivre le jeu avec attention, deux derrière la joueuse, la troisième au centre, derrière le bâton. L'interprétation de la scène est débattue. Le tirage au sort d'Aphrodite doit-il déterminer l'ordre dans lequel les jeunes filles se marieront, sur le modèle des bergers de Calpurnius Siculus? La joueuse est probablement la numphê, identifiée par le coffre qui lui sert de siège et évoque sa dot. La femme debout au centre, la tête couverte d'un voile qui la différencie de ses compagnes, pourrait avoir déjà passé par le klêros d'Aphrodite, assistant à l'épreuve de ses compagnes ${ }^{85}$. Notons que le fond du vase porte un motif de roue qui pourrait représenter une iunx.

Un oôn similaire, provenant probablement de la même tombe ${ }^{86}$, porte également une scène en rapport avec le mariage, celle d'un rapt, peut-être celui d'Hélène par Pâris. Un jeune homme court vers la gauche en tenant deux lances à la main. Il regarde derrière lui le quadrige conduit par un second personnage masculin qui enlace une jeune femme. Au-dessus du quadrige est peinte une couronne de laurier célébrant le succès de l'opération. Derrière le quadrige, Aphrodite, coiffée d'un haut diadème, un sceptre dans la main droite, s'appuie à un pilier. Derrière elle, Éros assis sur un rocher fait tourner une iunx pour lier le sort des amoureux.

31 L'enjeu nuptial du tirage au sort avec les doigts est traité de manière comique sur un canthare du Cabirion de Thèbes (Fig. 15 ; vers 420-410 av. J.-C.) ${ }^{87}$. La scène parodie le jugement de Pâris, appelé à choisir qui d'Athéna, Aphrodite et Héra est la plus belle des déesses. Sur une face, Héra attend la décision de Pâris aux côtés de Niké, qui tient déjà la couronne de la victorieuse, et d'Hermès qui a conduit Pâris sur le lieu du concours. Sur l'autre face, Pâris, assis sur un rocher, la tête coiffée d'un chapeau phrygien, joue de la lyre. Devant lui, deux divinités, sans doute Aphrodite et Athéna, sont assises et tiennent un bâton afin de tirer au sort l'issue du concours. Cependant, leurs mains placées aux extrémités du bâton pourrait indiquer qu'elles ont toutes deux gagné en même temps. Leur égalité serait une manière de dire combien le choix de Pâris est impossible.

n représentant l'épisode du jugement comme un tirage au sort, le peintre suggère aussi que ni la séduction, ni les promesses des déesses n'ont déterminé le choix du prince troyen. Sa décision était inscrite dans le plan divin, comme ses conséquences tragiques, l'enlèvement d'Hélène et la guerre de Troie. Cette mise en scène parodique participe aussi à la construction de la figure d'Hélène. Hélène n'avait pas uniquement la réputation d'être experte en art magique. Son rapport au jeu traduit aussi sa déviance par rapport au rôle attendu de la femme grecque. Dans la parodique Histoire nouvelle de Ptolémée Héphestion ${ }^{88}$, l'invention de la morra lui est attribuée :

[...] Hélène fut la première à imaginer de tirer au sort à l'aide des doigts et elle gagna en jouant aux sorts avec Alexandre ; elle serait la fille d'Aphrodite ${ }^{89}$.

Elle aurait même choisi le nom de sa fille en le jouant aux osselets avec Pâris : 
Hélène aurait eu une fille d'Alexandre ; ils étaient en désaccord sur le nom qu'on lui donnerait: lui voulait l'appeler Alexandra, elle voulait l'appeler Hélène ; Hélène l'emporta dans une partie d'osselets et l'enfant reçut le même nom que sa mère ${ }^{90}$.

En conclusion, si l'iconographie des pratiques ludiques nous emmène loin du jeu réel et de la reconstitution de son déroulement, elle participe pleinement à la construction d'une histoire de l'intime et du genre dans le monde grec. Les imagiers nous permettent d'accéder à d'autres règles, plus subtiles, celles des valeurs collectives et sociales qui régissent la vie émotionnelle des jeunes filles et des garçons. Les jeux examinés ici sont tous caractérisés par la mise en scène d'un mouvement qui visualise un monde intérieur sous le signe de l'incertitude et de l'inquiétude. Leur dynamique traduit la perception du sentiment amoureux comme une prise de risque, faite de hauts et de bas, comme les balles, pommes ou pelotes de la jongleuse, aléatoire, comme le mouvement de la toupie, et imprévisible, comme le sort tiré avec les doigts. Le jeu manifeste ainsi à la fois l'anxiété et la volonté d'agir sur l'avenir. D'autres jeux d'adresse véhiculent cette tension, tels les osselets, comparés par Anacréon aux « folies et désordres d'Amour $\aleph^{91}$. La dimension métaphorique de ces images révèle aussi une façon active de penser le rôle des jeunes filles. Elles sont dépeintes expertes en joutes qui diffèrent de celles des garçons et mettent en scène leur adresse, comme dans le jeu

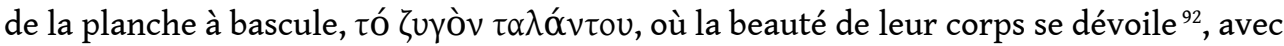

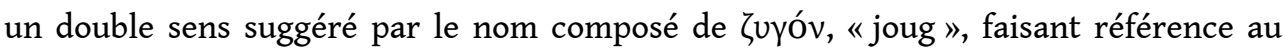
contexte nuptial. L'agôn érotique révèle que les femmes ne sont pas perçues comme de simples objets du désir masculin. Les imagiers les placent sur le plan d'un «jouer » où elles se projettent dans l'avenir ${ }^{93}$. Elles sont pensées discrètement actives, agissant sur l'indéterminé que concentre le champ sémantique du verbe paizô, la séduction, l'amour, la prospérité et le bonheur, sans oublier le plaisir, promesse de fécondité.

\section{Abréviations}

\begin{tabular}{|c|c|}
\hline$A B V$ & J. D. BEAZLEY, Attic Black-Figure Vase-Painters, Oxford, 1956. \\
\hline$A R V^{2}$ & J.D. BEAZLEY, Attic Red-Figure Vase-Painters, 2 e éd, Oxford, 1963. \\
\hline BA & $\begin{array}{l}\text { Beazley Archive, base de données en ligne: www.beazley.ox.as.uk/databases/ } \\
\text { pottery.htm. }\end{array}$ \\
\hline Paralipomena & $\begin{array}{l}\text { J.D. BEAZLEY, Paralipomena: Additions to 'Attic Black-figure Vase-painters' and 'Attic Red- } \\
\text { figure Vase-painters', Oxford, } 1971 \text {. }\end{array}$ \\
\hline$P G M$ & $\begin{array}{l}\text { K. PREISENDANZ (dir.), Papyri Graecae Magicae, } 2 \text { vols, } 2^{\mathrm{e}} \text { éd. revue par A. HENRICHS, } \\
\text { Stuttgart, Teubner, 1973-1974 (trad. anglaise : H.D. BETZ, The Greek Magical Papyri in } \\
\text { Translation, Including the Demotic Spells, Chicago, } 1992,2^{\mathrm{e}} \text { éd.). }\end{array}$ \\
\hline
\end{tabular}



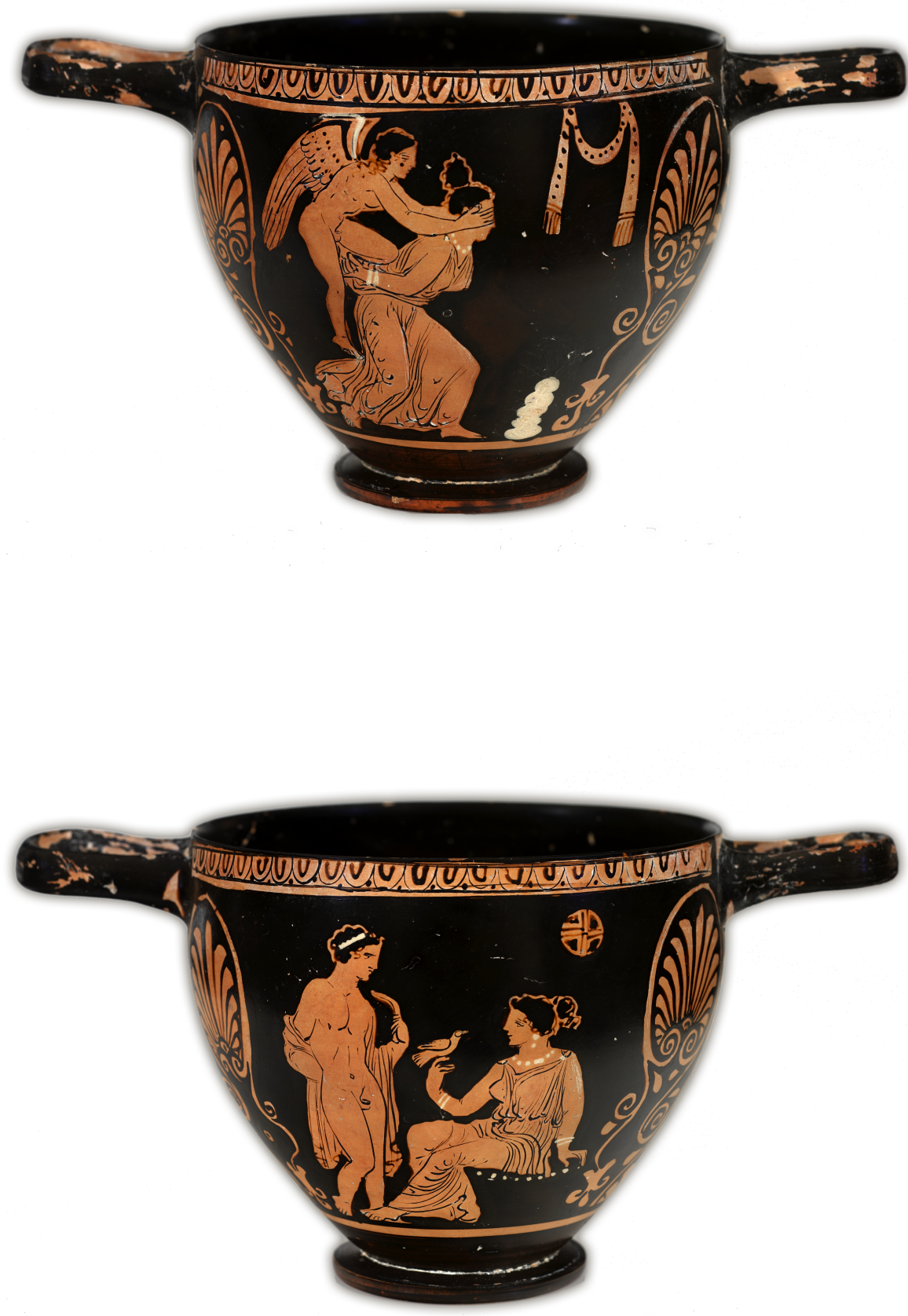

FIg. 1.SKYPHOS APULIEN, Vers 375-350 AV. J.-C. MUSEUM OF ART, Rhode Island School of Design 25-089.

Photo Erik Gould, courtesy of the Museum of Art, Rhode Island School of Design, Providence. 


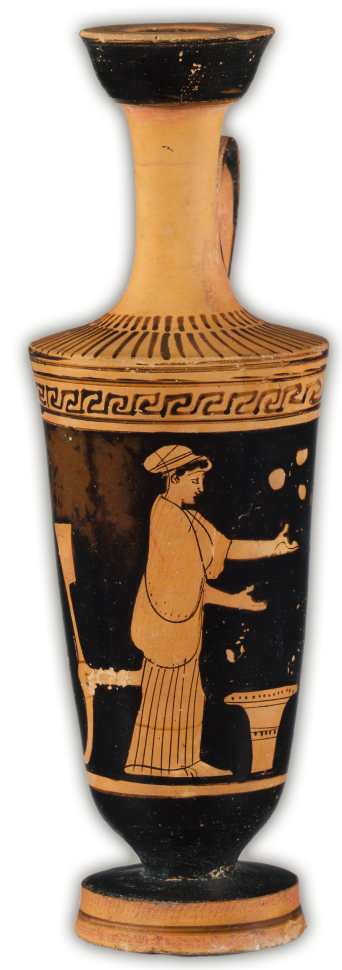

Fig. 2.Lécythe attique, vers 480-470 av. J.-C. New York, Metropolitan Museum of Art 41.162.147. Rogers Fund, 1941.

Photo du musée.

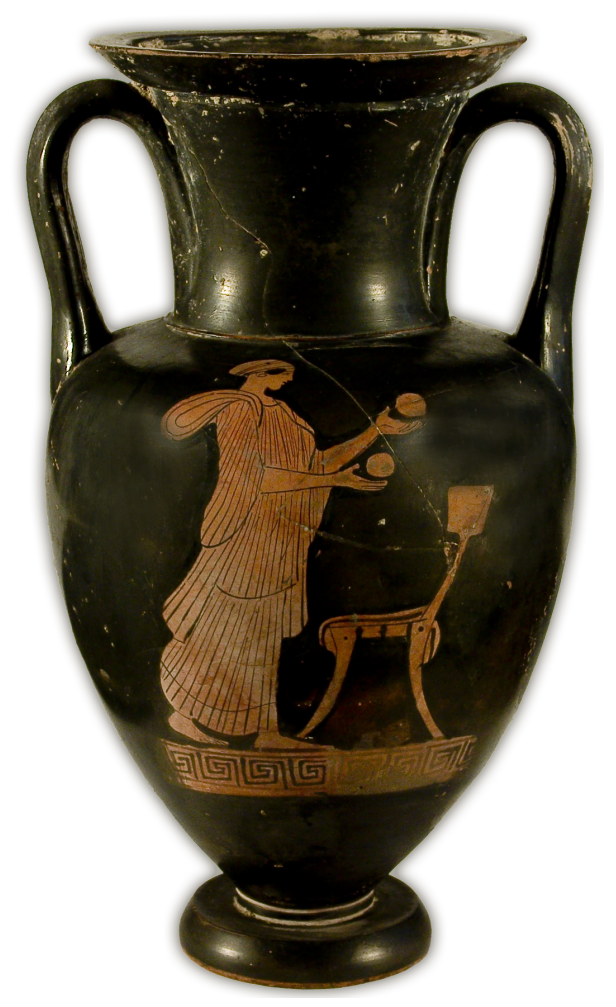




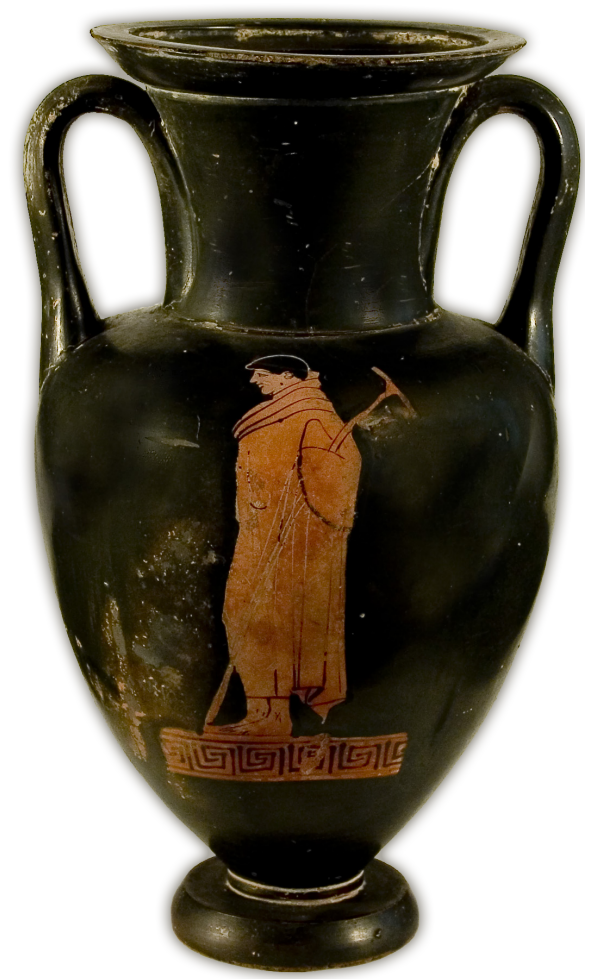

Fig. 3.Amphore attique, milieu du ve s. av. J.-C. Milan, coll. Sambon, inv. St. 1323 ; Sambon 14 ; Scala 698

(c) Milan, Soprintendenza per i Beni Archeologici della Lombardia.

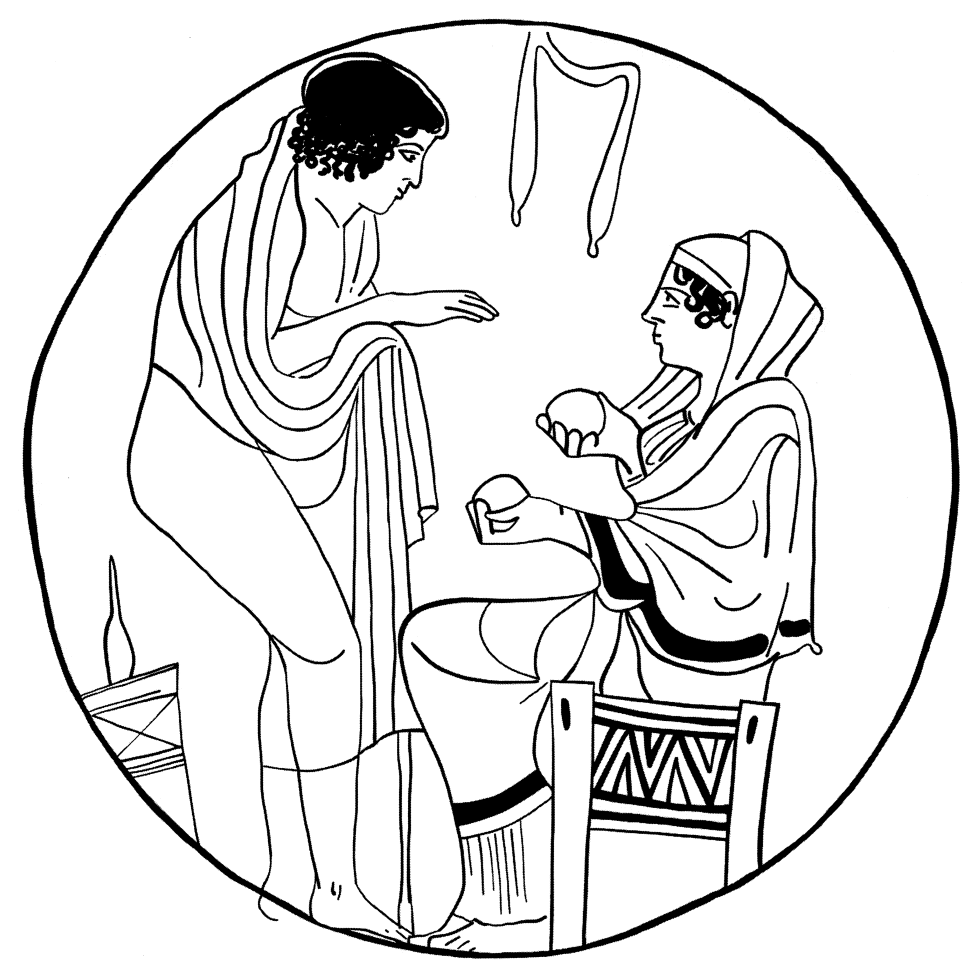

Fig. 4.Coupe attique, vers 460 av. J.-C. Boston, Museum of Fine Arts. Dessin V. Dasen 


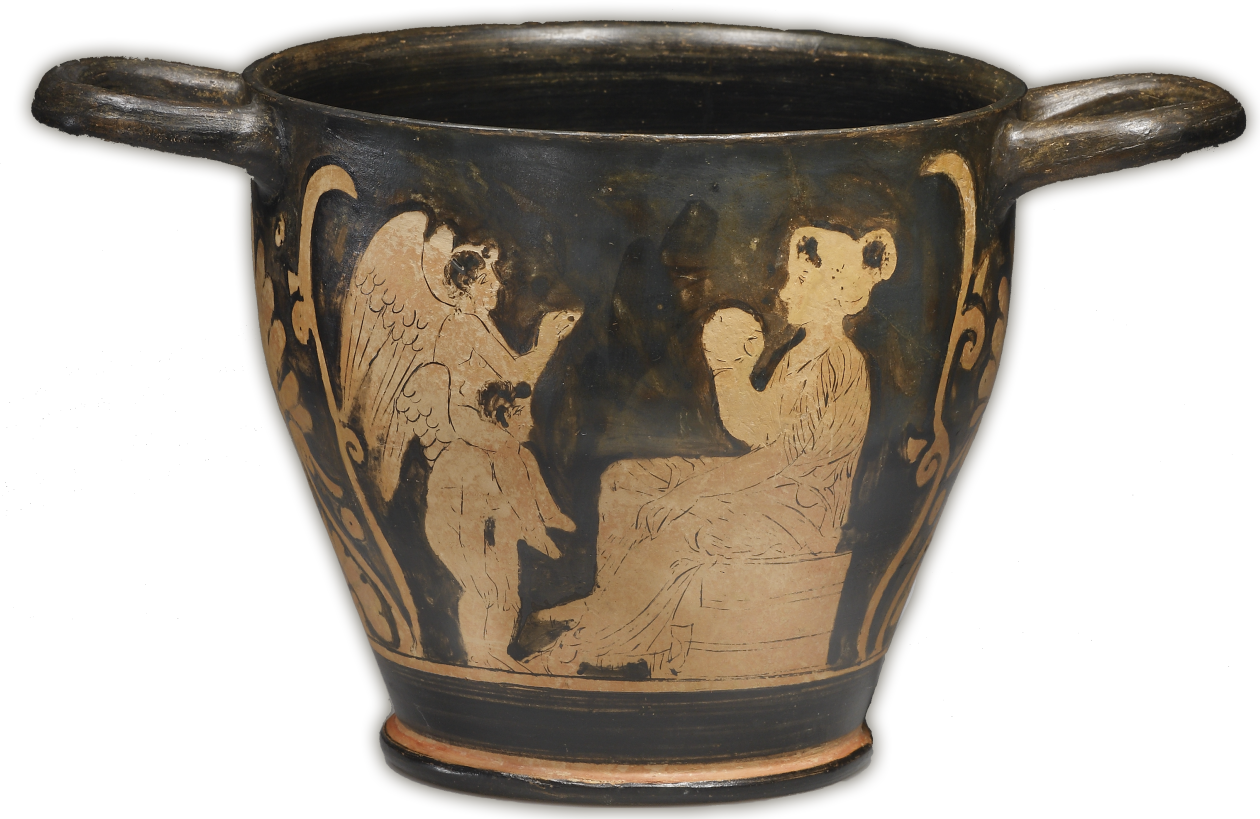

Fig. 5.Skyphos béotien, dernier quart du ve s. av. J.-C. Collection privée

(c) Photographie Nik Bürgin, Bâle 2014 ; courtoisie de la Galerie Jean-David Cahn AG, Bâle.

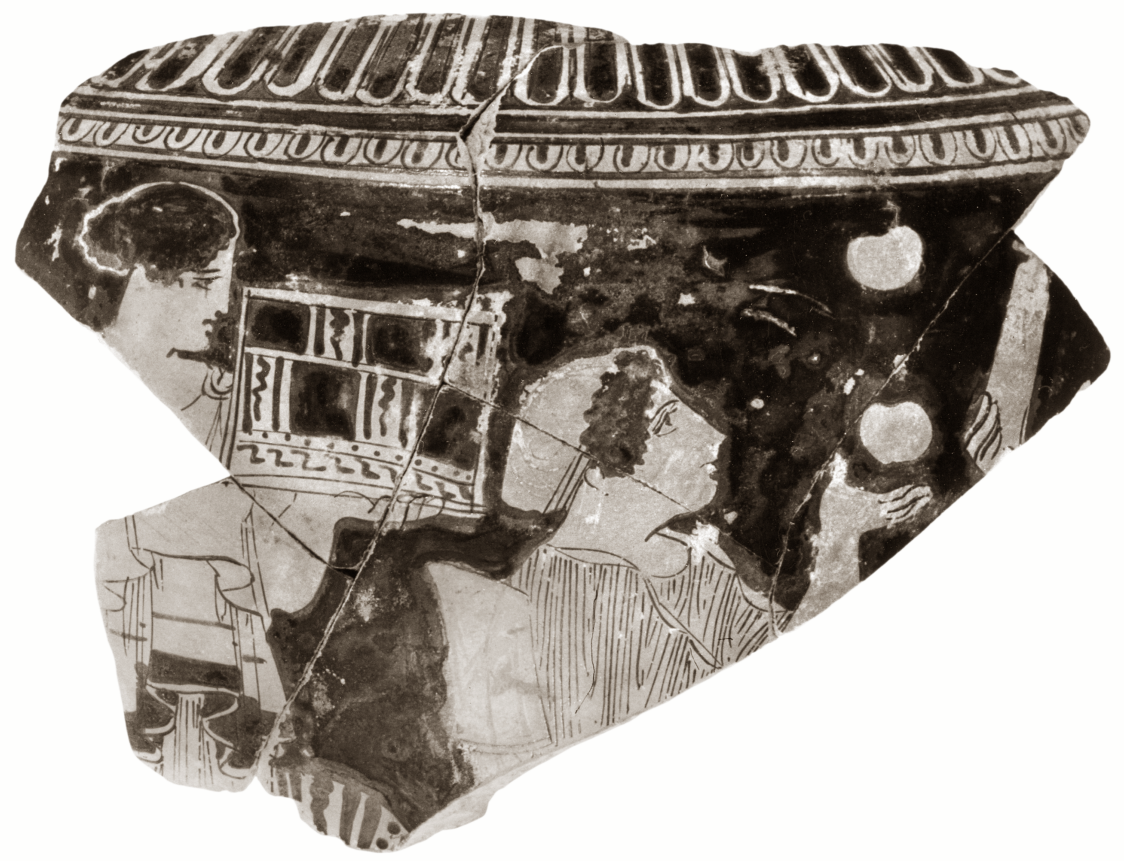

Fig. 6.Lébès gamikos attique, vers $430-420$ av. J.-C. Tubingen, Eberhard-Karls-Universität, Archäologisches Institut 5643.

Photo Thomas Zachmann. 


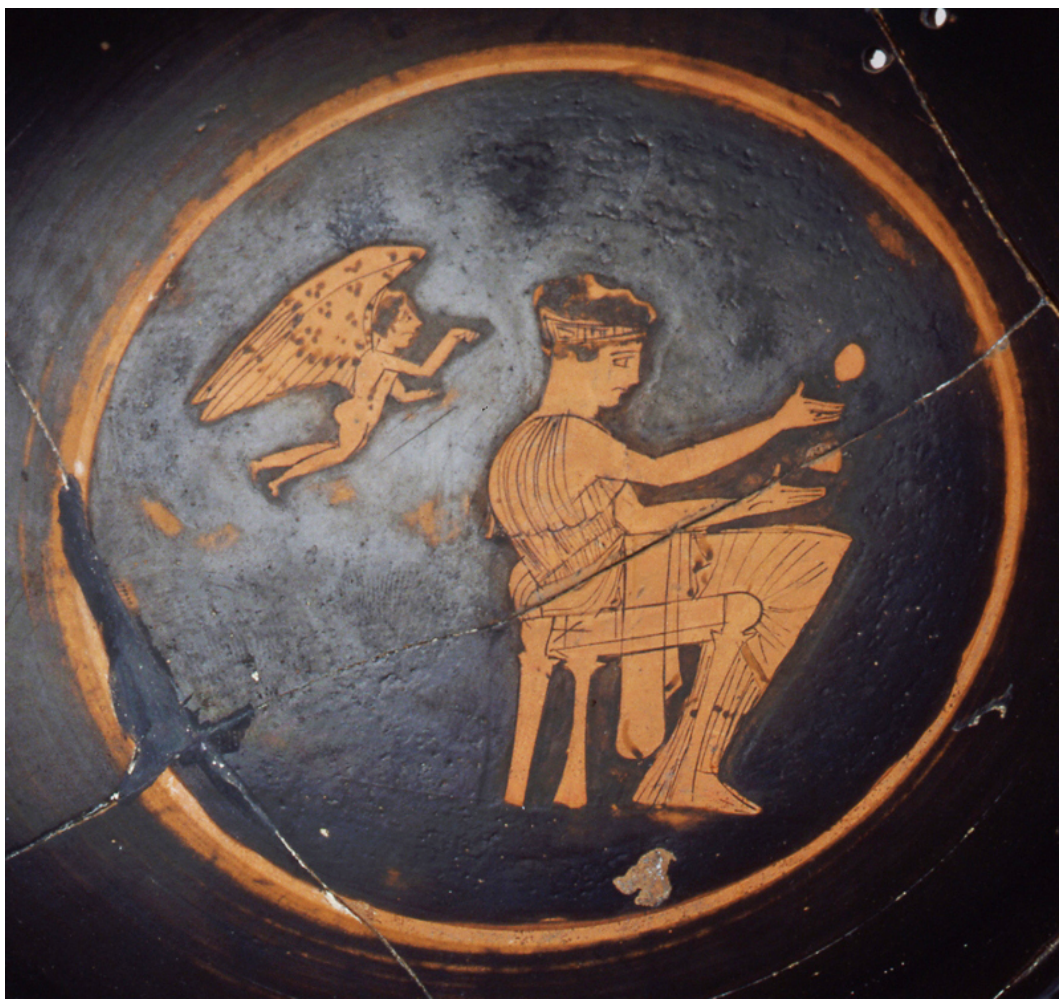

Fig. 7.Coupe attique, 440-420 av. J.-C. University of Chicago, the David and Alfred Smart Museum of Art, 1967.115.347. The F.B. Tarbell Collection, Gift of E.P. Warren, 1902.

(C) 2016 courtesy of the David and Alfred Smart Museum of Art, The University of Chicago.

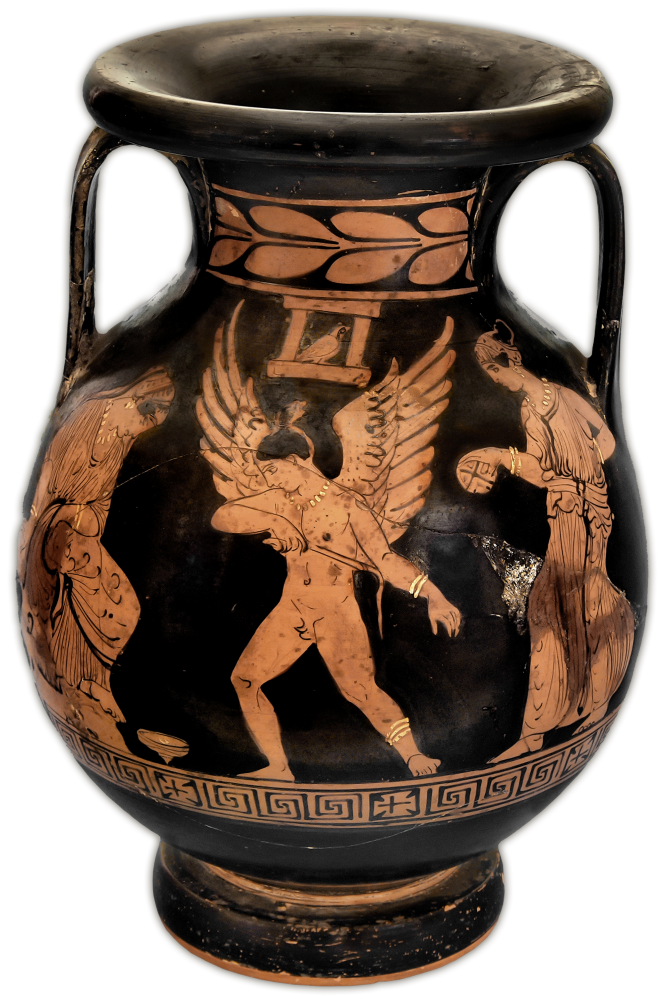




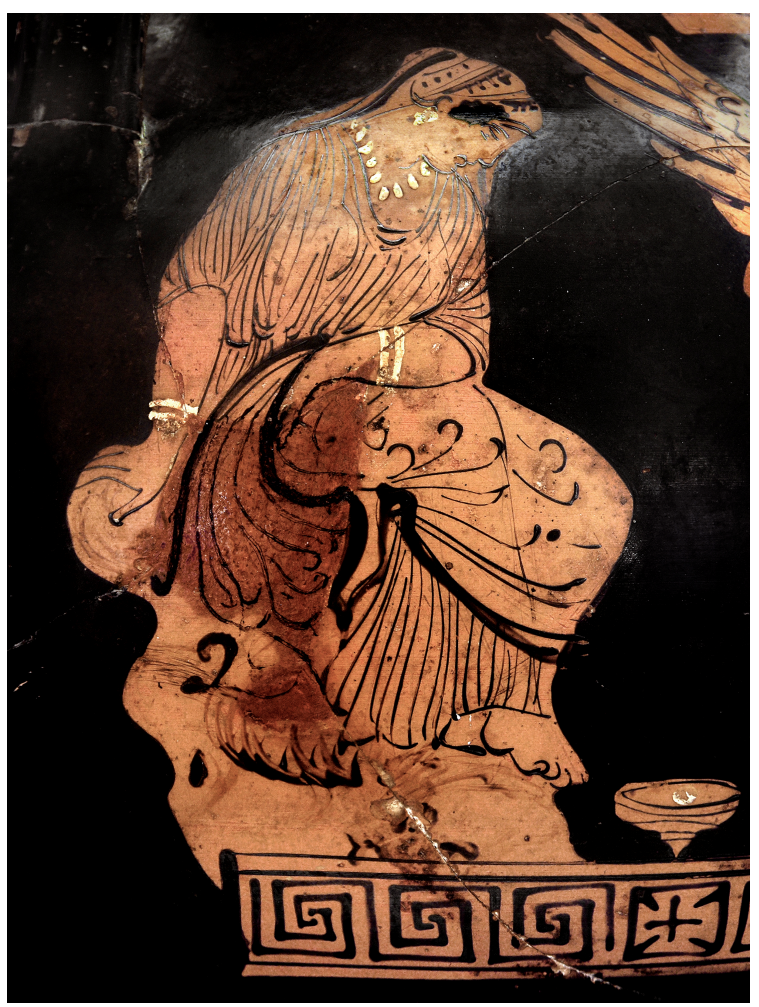

Fig. 8.Péliké apulienne, 390-380 av. J.-C. Matera, Museo Nazionale Ridola 14924. Su concessione del Ministero dei Beni e della Attività Culturali e del Turismo

Soprintendenza Archeologia della Basilicata.

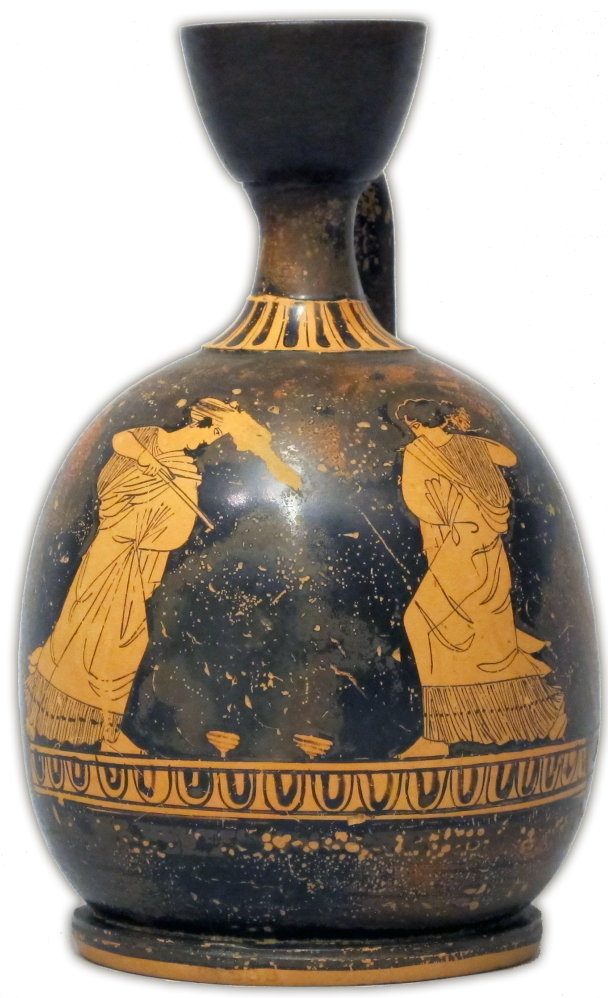

Fig. 9.Lécythe attique, 440-430 av. J.-C. New York, Metropolitan Museum of Art 1875, 75.2.9. Gift of Samuel G. Ward, 1875.

Photo du musée. 


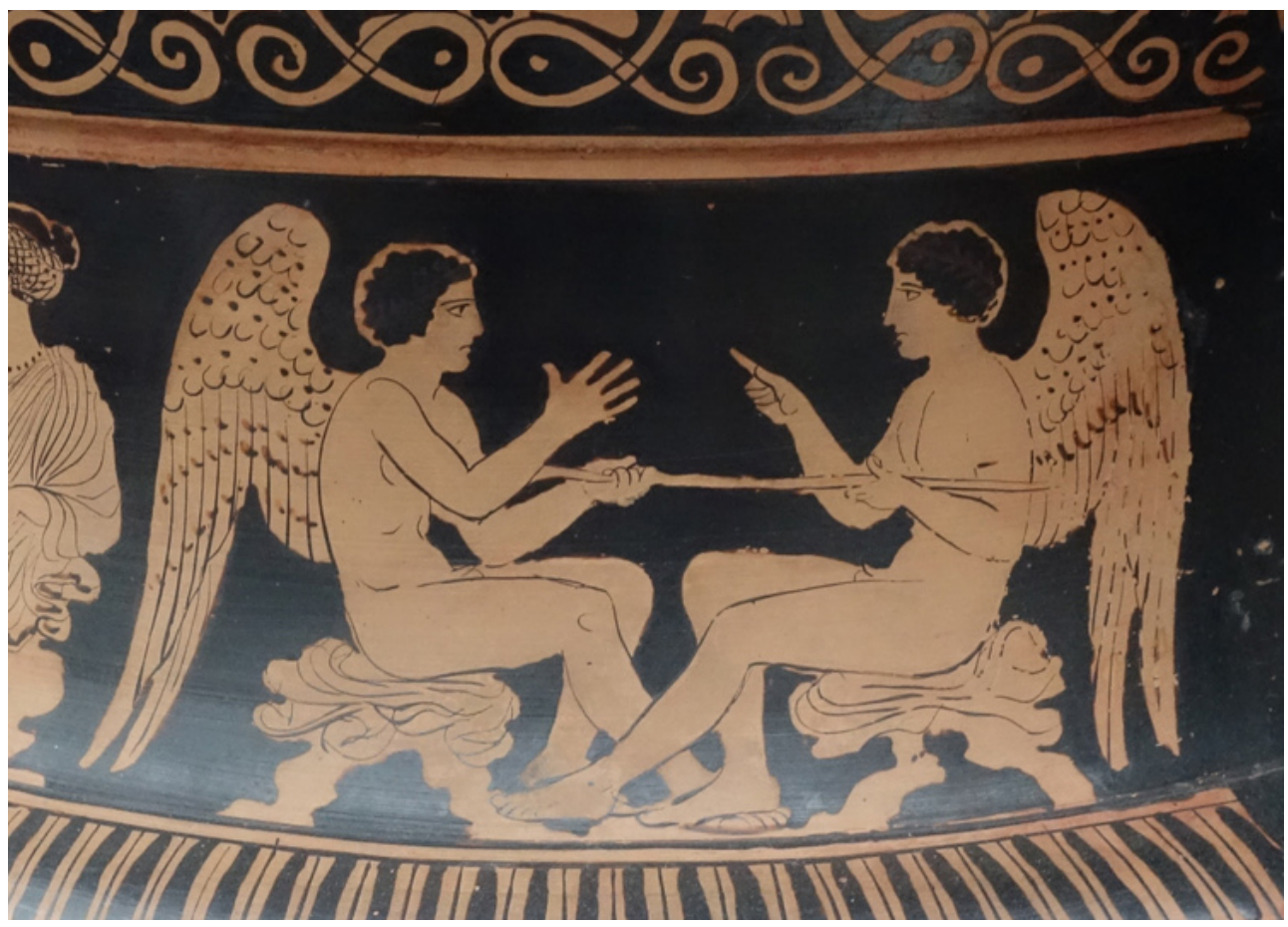

Fig. 10.Cratère apulien, vers 420 av. J.-C. Munich, Antikensammlung 3268.

Photo V. Dasen.

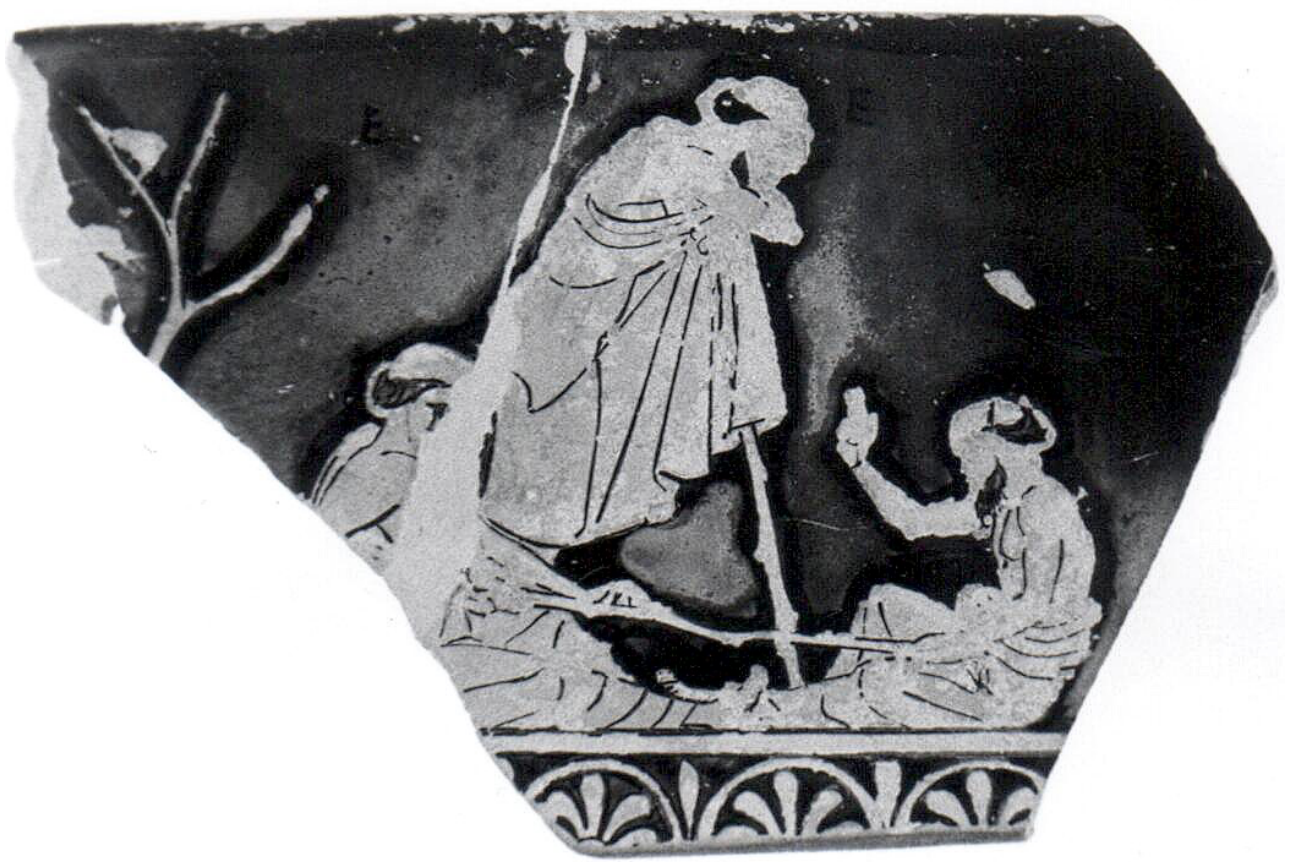

Fig. 11.Fragment de coupe attique, vers 450 av. J.-C. Bâle, Coll. Cahn HC 431. Photo A. Lezzi-Hafter. 


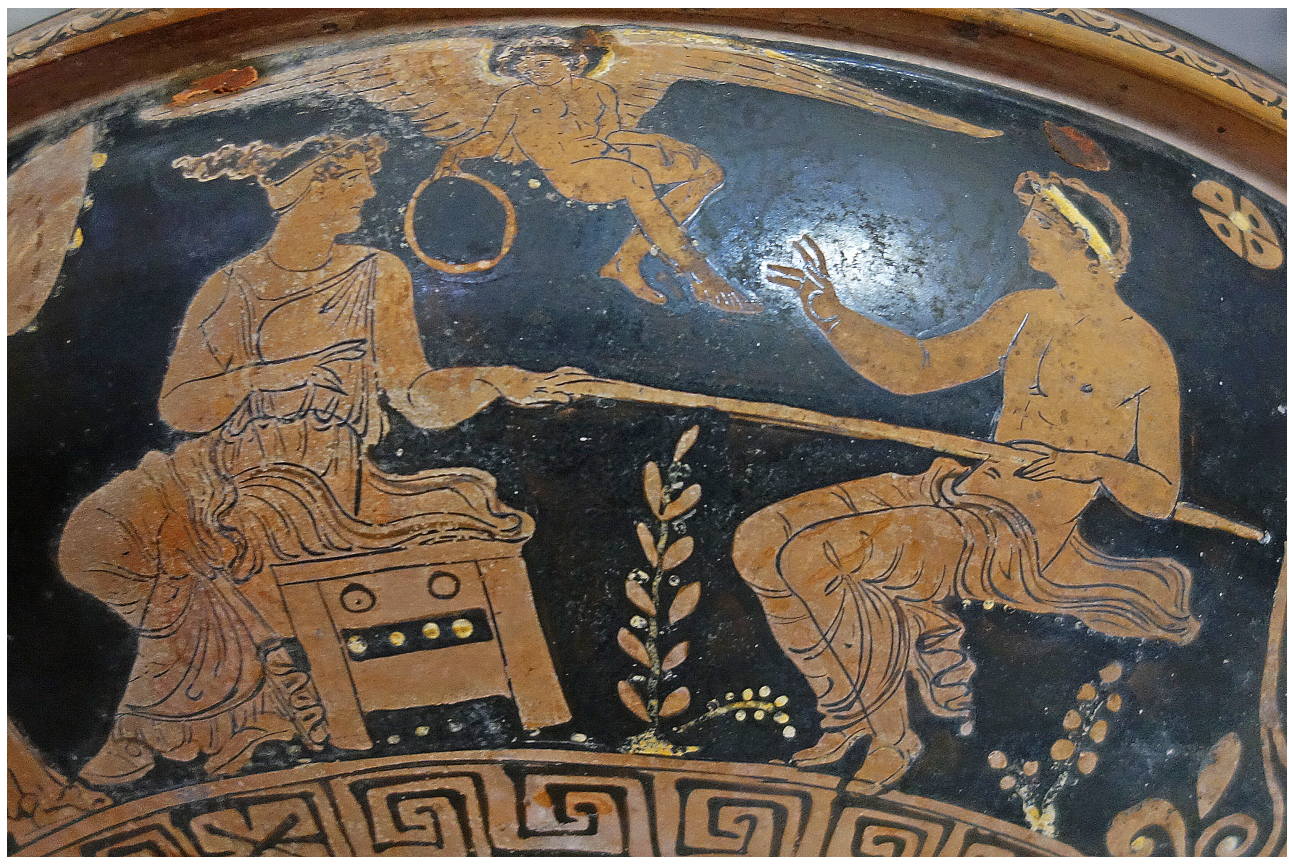

Fig. 12.Lékané apulienne, vers 380 av. J.-C. Naples, Musée archéologique 82038, H 2574. Photo V. Dasen.

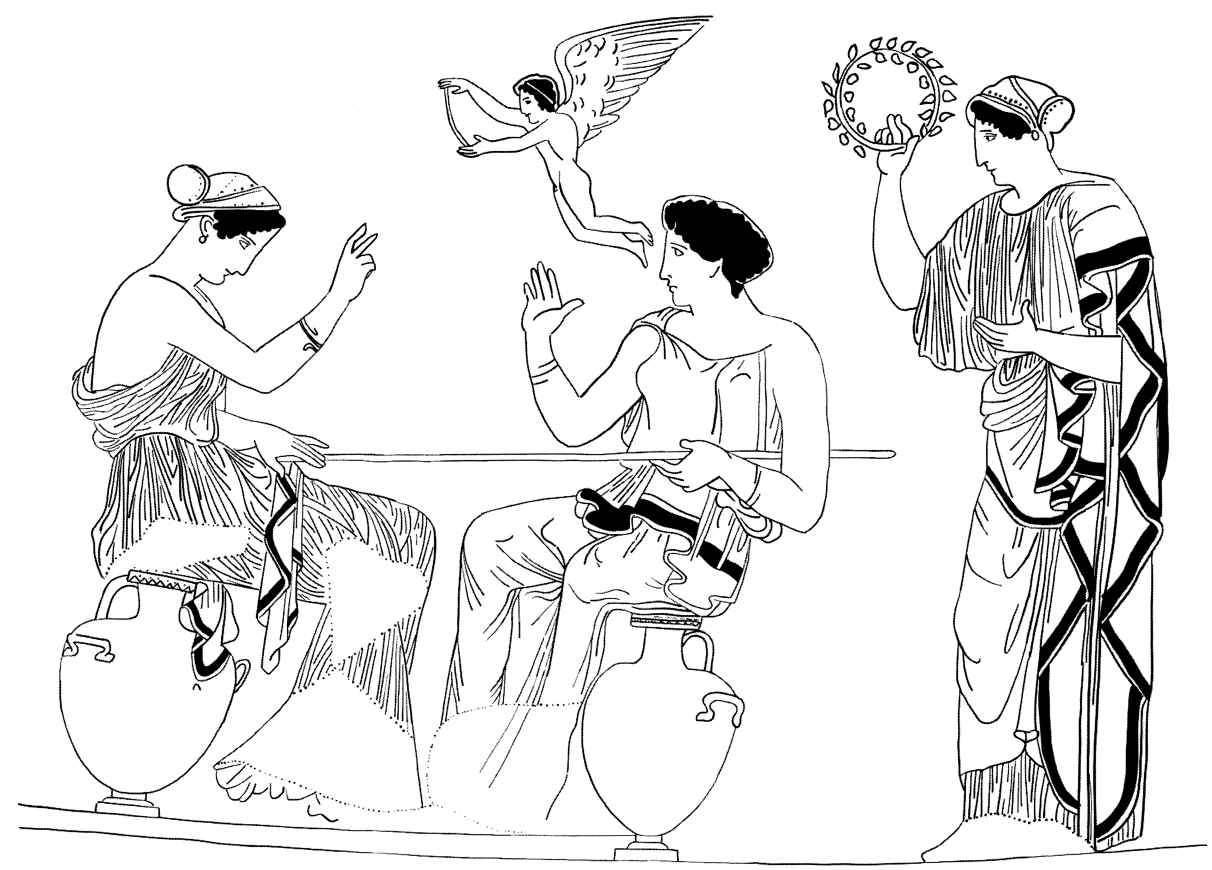

Fig. 13.Hydrie attique, vers 440-420 av. J.-C. Goluchow, Musée archéologique national 14299.3. Dessin V. Dasen. 


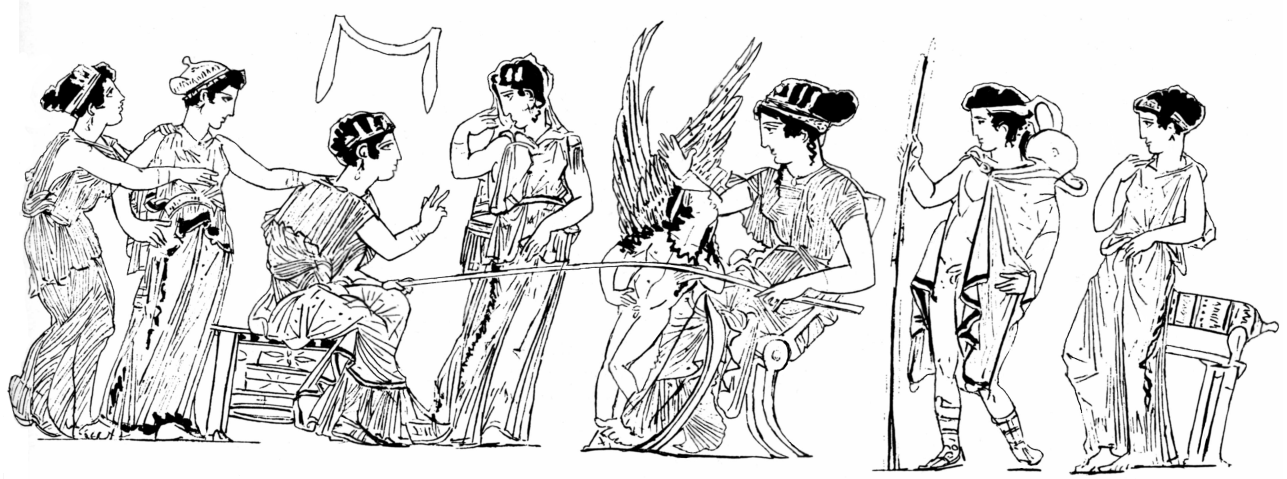

Fig. 14. Oôn attique, vers 420 av. J.-C., Athènes, Musée archéologique national 332.

Dessin d'après H. Metzger.
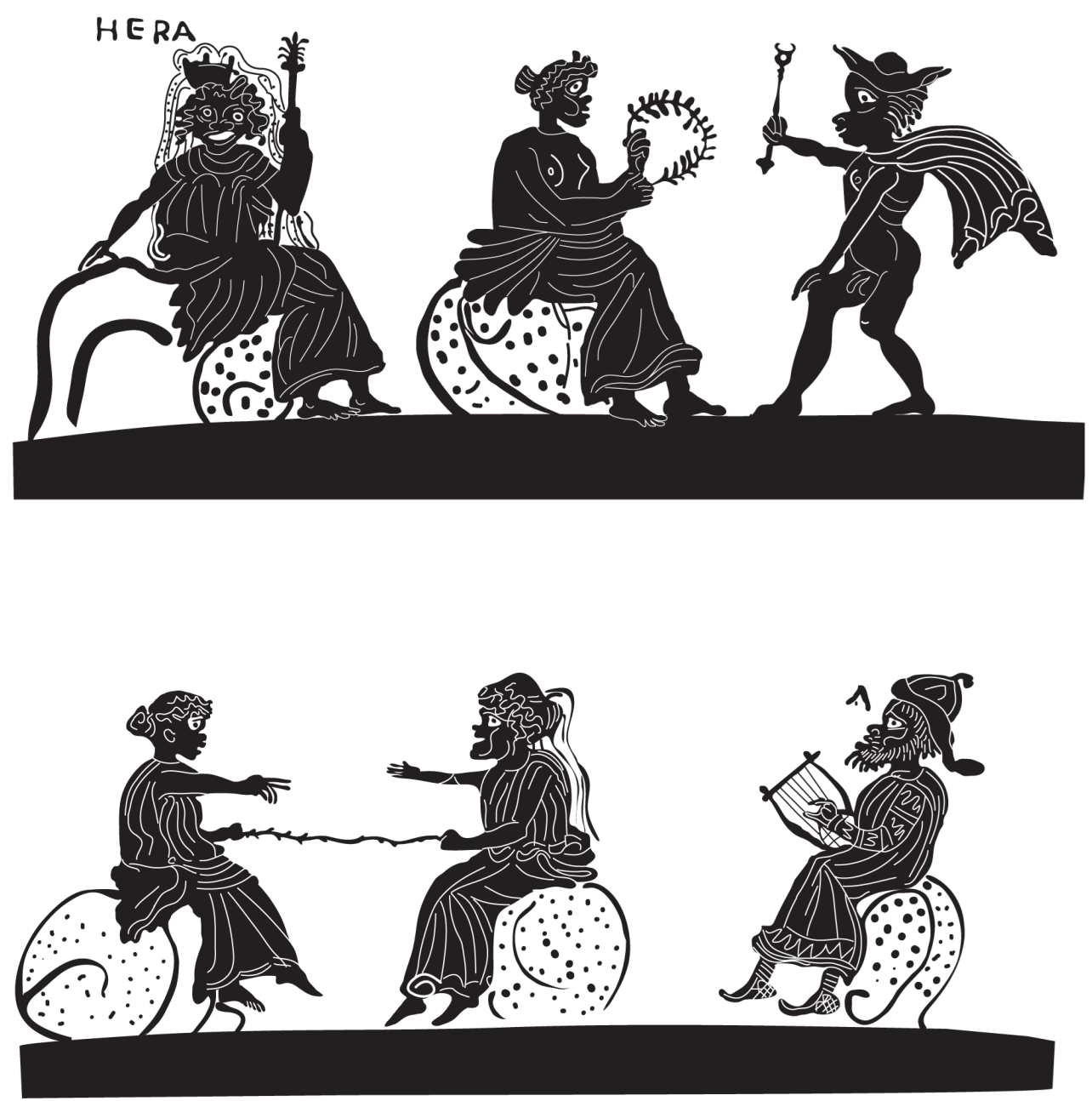

(c) Alexandre G. Mitchell

Fig. 15. Skyphos béotien, vers 420-410 av. J.-C., Boston, Museum of Fine Arts 99.533. Dessin A.G. Mitchell. 


\section{NOTES}

1. Pollux, Onomasticon IX, 119 : « les joueurs visent à distance une pierre qu'ils ont dressée ; celui qui n'est pas arrivé à la renverser porte sur son dos celui qui y est arrivé ; ce dernier lui cache les yeux jusqu'à ce qu'il atteigne sans dévier la pierre appelée Dioros ('séparation')» (trad. A.-L. Rey). Ch. SCHEFFER, "Return or no Return: The so-Called Ephedrismos Group and the Hephaisteion ", OAth 21 (1996), p. 169-188; U. MANDEL, «Die ungleichen Spielerinnen. Zur Bedeutung weiblicher Ephedrismosgruppen », in P.C. BOL (éd.), Hellenistische Gruppen. Gedenkschrift für Andreas Linfert, Mainz, 1999, p. 213-266; A. SCHMÖDER-VEIT, ",Huckepack' - Aphrodite treibt ihr Spiel », in R. wÜNSCHE, F. KNAUSS, Lockender Lorbeer. Sport und Spiel in der Antike, Ausstellung Staatliche Antikensammmlungen München, München, 2004, p. 426-430.

2. .Rhode Island School of Design 25.089, atelier du P. de l'Ilioupersis ; CVA USA2, RISD 1, 1933, p. 39, pl. 28, 2 a-c ; A.H. ASHMEAD, K.M. PHILIPS, Jr. Classical Vases, Museum of Art, The Rhode Island School of Design, Providence, 1976, p. 50-51, fig. 59 ; A.D. TRENDALL, A. CAMBITOGLOU, The Red-Figured Vases of Apulia, 1978, p. 208, 136; M. REHO-BUMBALOVA, «Eros e il gioco dell'ephedrismos su una lekythos di Sofia », BABesch 56 (1981), p. 153-156, fig. 5 ; SCHEFFER, o.c. (n. 1), fig. 6 ; V. DASEN, U. SCHÄDLER, «Introduction. Jeux et jouets dans l'Antiquité », Archéothema. Jeux et jouets gréco-romains 31 (2013), p. 4-6.

3. .Euripide, Hippolyte, 546 (trad. L. Méridier, CUF). Aristote, Histoire des animaux, VI, 18, 572 a 30 utilise le verbe paizô pour désigner les juments en rut qui « folâtrent » entre elles. Sur le mariage

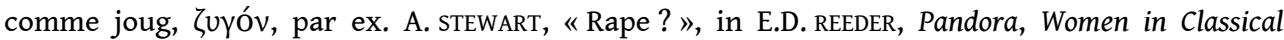
Greece, Baltimore/Princeton, 1995, p. 74-90 ; P. BRULÉ, « Des osselets et des tambourins pour Artémis », in La Grèce d'à côté. Réel et imaginaire en Grèce antique, Rennes, 2007, p. 81-83 (sur les anthroponymes féminin en rapport avec le cheval); C. CALAME, Les chœurs de jeunes filles en Grèce archaïque, Rome, I, 1977, p. 414-415 ; id., L'Éros dans la Grèce antique, Paris, 1996, p. 164-167 (avec la bibliographie antérieure).

4. .Cf. les offrandes à Trézène de ceintures à Athéna Apatouria avant le mariage, et l'image de la ceinture dénouée par l'époux pour signifier l'union sexuelle ; P. SCHMITT, « Athéna Apatouria et la ceinture. Les aspects féminins des Apatouries à Athènes ", Annales (ESC) 6 (1977), p. 1059-1073.

5. .Cf. V. CELLE, « La femme et l'oiseau dans l'iconographie grecque », Pallas 42 (1995) p. 113-128. L'oiseau peut aussi indiquer une classe d'âge. Sur l'association du motif de l'oiseau aux individus pré-adultes, A. COHEN, «Introduction: Childhood between Past and Present », in A. COHEN, J. RUTTER ( éd.), Constructions of Childhood in Ancient Greece and Italy, Princeton, 2007, p. 15-20.

6. Coll. privée, Bari; information de A.D. Trendall; ASHMEAD, PHILIPS, o.c. (n. 2), p. 51. Sur l'iconographie du bain prénuptial, voir par ex. la pyxis, New York, MMA 1972.118.148 ; J.H. OAKLEY, R.H. SINOS, The Wedding in Ancient Athens, Madison, 1993, p. 16, fig. 20-21 ; F. LISSARRAGUE, « Regards sur le mariage grec", in O. CAVALIER (dir.), Silence et fureur, la femme et le mariage en Grèce: les antiquités grecques du Musée Calvet, Avignon, 1996, p. $422-423$ (fig.).

7. .Dans un autre registre, la décision divine peut être exprimée visuellement par le motif de l'érostosasie, la pesée des Érotes sur une balance tenue par Aphrodite, par ex. sur une bague en or, Boston, Museum of Fine Arts, 23.593, vers 450-425 av. J.-C. ; J. BOARDMAN, Greek Gems and Finger Rings, London, 2001, p. 216, pl. 666. Sur le jeu de la planche à bascule, dont la signification est proche de celle de la balance des Érotes, voir aussi plus bas n. 92.

8. .Voir l'étude iconographique accompagnée d'un catalogue de S. PFISTERER-HAAS, « Mädchen und Frauen im Obstgarten und beim Ballspiel, Untersuchungen zu zwei vorhochzeitlichen Motiven und zur Liebessymbolik des Apfels auf Vasen archaischer und klassischer Zeit ", MDAI(A) 118 (2003), p. 139-195. 
9. Notons que l'espace du verger est déjà érotisé par la présence d'un oiseau-phallus sur un lécythe à figures noires (vers 500-475 av. J.-C.) ; Athènes, Musée national 471; ABV 502, 100 ; BA 305429 ; J. BOARDMAN, « The Phallos-Bird in Archaic and Classical Greek Art », RA (1992), p. 230, fig. 7 ; PFISTERER-HAAS, o.c. (n. 8), p. 149, cat. SO 13, fig. 2 (oiseau phallus), et p.164-165, cat. FF4, pl. 40, 1 (verger et panier à laine).

10. .New York, Metropolitan Museum of Art 41.162.147 ; ARV 22 733,66 ; BA 209053 ; PFISTERER-HAAS, o.c. (n. 8), p. 169, cat. J 12. Cf. le lécythe à fond blanc, Université de Leipzig T 429, vers 460-450 av. J.-C. ; $A R V^{2}$ 10,701; D. vanHove (éd.), Le sport dans la Grèce antique. Du jeu à la compétition, Bruxelles, 1992, p. 165-166, cat. 20.

11. Bruxelles, Bibliothèque royale 9 ; $A R V^{2}$ 863,31; BA 211359 ; PFISTERER-HAAS, o.c. (n. 8), p. 170, cat. J 29, pl. $42,5$.

12. .Cf. la numphê kalê dans la même pose sur un alabastre conservé à Paris, Cabinet des Médailles 508, vers 470 av. J.-C. ; ARV ${ }^{2} 1610$; BA 21648 ; F. LISSARRAGUE, « Femmes au figuré », in P. SCHMITT PANTEL (dir.), Histoire des femmes en Occident, I, L'Antiquité, Paris, 1991, p. 182, fig. 12. Voir aussi la jongleuse debout avec trois balles, sans kalathos, sur deux lécythes attiques à figures rouges, vers 450/420 av. J.-C. ; WÜNSCHE, KNAUSS, o.c. (n. 1), p. 423, cat. 238-239, fig. 46, 2-3 ; PFISTERER-HAAS, o.c. (n. 8), p. 169, cat. J 15, pl. 41, 5-6.

13. .Oxford, Ashmolean Museum, 1965.130 ; ARV 2864,15 ; BA 211377 ; PFISTERER-HAAS, o.c. (n. 8), p. 170 , cat. J 30 , pl. 43,1 .

14. .Munich, Antikensammlung 2676 ; $A R V^{2} 393,27$; BA 204225 ; F. LISSARRAGUE, « Women, Boxes, Containers: some Signs and Metaphors ", in REEDER o.c (n. 3), p. 95, fig. 5 ; PFISTERER-HAAS, o.c. (n. 8), p. 170 , cat. J 34, pl. 42, 1 ; wÜNSCHE, KNAUSS, o.c. (n. 1), p. 423, cat. 240, fig. 46.4.

15. .Milan, coll. Sambon St. 1323 ; Sambon 14 ; Scala 698 ; ARV ${ }^{2}$ 670,5, Manière du P. de Londres E 342 ; C. LAMBRUGO, "Anfora nolana antica a figure rosse ", in A. CERESA MORI, C. LAMBRUGO, F. SLAVAzzi (éd.), L'infanzia e il gioco nel mondo antico. Materiali della Collezione Sambon di Milano, Milano, 2012, p. 84.

16. .Boston, Museum of Fine Arts 13.84 ; $A R V^{2} 883,61$; BA 211626 ; PFISTERER-HAAS, o.c. (n. 8), p. 172, pl. 44,1 .

17. Odyssée VI, 110-142.

18. .V. PIRENNE-DELFORGE, L'Aphrodite grecque, Athènes/Liège, 1994 (Kernos, suppl. 4), p. 410-412; D. FASCIANO, "La pomme dans la mythologie gréco-romaine », in M. FORTIN et al. (dir.), Mélanges d'études anciennes offerts à Maurice Lebel, Québec, 1980, p. 45-55. Sur la pomme d'Éris, I. RAAB, Zu den Darstellungen des Parisurteils in der griechischen Kunst, Bern, 1972, p. 52-60 ; F. GHERCHANOC, Concours de beauté et beautés du corps en Grèce ancienne. Discours et pratiques, Paris, 2015, p. 49-64.

19. Sur les différentes versions relatives au kêpos des Hespérides et d'Aphrodite, CALAME 1996, o.c. (n. 3), p. 216-219.

20. .Sur le lien entre les scènes de cueillettes et le mariage sur les pinakes de Locres, voir par ex. Chr. sourvinou-INwood, «Persephone and Aphrodite at Locri: A Model for Personality Definitions in Greek Religion », JHS 98 (1978), p. 101-121, spéc. p. 108-109 sur les offrandes de modèles en terre cuite de balles et de grenades; EAD., «Due protettrici della donna a Locri Epizefirii: Persefone e Afrodite », in G. ARRIGONI (éd.), Le donne in Grecia, Roma/Bari, 1985, p. 203-221.

21. .Daphnis et Chloé III, 34, 2 - 34, 3.

22. .Par ex. Aristophane, Assemblée des femmes, 903 et Lysistrata, 155. Voir les sources réunies par A.R. LITTLEWOoD, «The Symbolism of the Apple in Greek and Roman Literature », HSPh, 72 (1968), p. 147-181; D.E. GERBER, "The Female Breast in Greek Erotic Literature », Arethusa 11 (1978), p. 203-212. Sur la métaphore clitoridienne, J.J. WINKLER, Désir et contraintes en Grèce ancienne (trad. S. Boehringer, N. Picard), Paris, 2005, p. 344-345. Sur le rôle des pommes ou coings dans les rites de mariage, M. DETIENNE, Dionysos mis à mort, Paris, 1977, p. 103-104 ; OAKLEY, SINOS, o.c. (n. 6), p. 35.

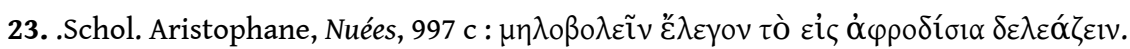


24. .Théocrite, Idylles V, 88-89.

25. .Aristophane, Nuées, 996-999.

26. .Anthologie Palatine V, 80. Voir aussi V, 79.

27. .Philostrate, La galerie de tableaux I, 6, 3.

28. .Anthologie Palatine V, 214. K. GUTZWILLER, «Images poétiques et réminiscences artistiques dans les épigrammes de Méléagre ", in É. PRIOUX, A. ROUVERET (dir.), Métamorphoses du regard ancien, Paris, 2010, p. 67-112.

29. .Boston, Museum of fine arts 95.26 ; $A R V^{2} 1317,3$; BA 220527 ; PFISTERER-HAAS, o.c. (n. 8), p. 156, cat. RO 11, pl. 39, 1. Voir aussi la kalpis béotienne (400-390 av. J.-C.), ibid., fig. 3 (Hélène cueille les fruits en présence de Thésée et d'Aphrodite).

30. Théocrite, Idylles III, 40-42. Chez Hésiode et Apollodore, Hippomène les jette sur la piste. Sur

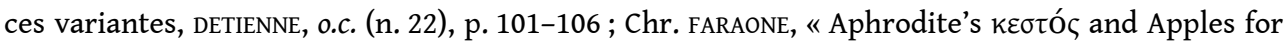
Atalanta: Aphrodisiacs in Early Greek Myth and Ritual », Phoenix 44 (1990), p. 219-243.

31. .Voir la variante d'Antoninus Liberalis, Métamorphoses I (Ctésylla et Hermocharès).

32. .Ovide, Héroïdes XXI, 124-125: «Une pomme a pris Cydippe, une pomme a pris la fille de Schoeneus. »

33. .Cf. WiNKLER, o.c. (n. 22), p. 143-196, sur l'usage d'agôgai pour soumettre le désir féminin au contrôle masculin.

34. .PGM CXXII ; FARAONE, o.c. (n. 30), p. 233-235 ; G. FICHEUX, Eros et Psyché: l'être et le désir dans la magie amoureuse antique, Université Rennes 2, 2007, thèse de doctorat, inédite, en accès libre $<$ tel-00189672v2>, p. 83 ; des recettes aphrodisiaques utilisent des parties de la pomme, comme les pépins. Cf. la consommation de la pomme ou coing lors des rites de mariage, à l'image du pépin de grenade croqué par Perséphone et qui la lie pour toujours à Hadès; Hymne homérique à Déméter, 372-374, 411-413 ; DETIENNE, o.c. (n. 22), p. 104.

35. Collection privée; Cahn Auktionen AG 9 (2014), $\mathrm{n}^{\circ}$ 132. Je remercie F. Lissarrague de m'avoir signalé ce vase.

36. .Cf. l'offrande de la balle de Timareta à Artémis Limnatis à la veille du mariage ; Anthologie Palatine VI, 280 ; BRULÉ 2007, o.c. (n. 3), p. 69-83. Les Arrhéphores ont des jeux de balles près du sanctuaire d'Aphrodite sur l'Acropole ; Plutarque, Vie d'Isocrate, 836c. BRULÉ 1987, o.c. (n. 3), p. 9091. Cf. Aphrodite en compagnie de deux jeunes filles, dont l'une lance une balle sur un fragment d'hydrie attique du Peintre de Kléophon (vers 440-430 av.J.-C.); Tubingen, Archäologisches Institut 1632 ; BA 215201 ; R. ROSENZWEIG, Worshipping Aphrodite, Art and Cult in Classical Athens, Ann Arbor, 2004, p. 49-51, fig. 20.

37. .Oxford, Ashmolean Museum 1890.27 ; Paralipomena 217.2 ; BA 380847 ; VANHOVE, o.c. (n. 10), p. 169, cat. 22. L'entraîneur est face à trois équipes de joueurs sur l'amphore du Peintre de la balançoire; Londres, British Museum B 182; ABV 306,42; BA 301522 ; CVA London, British Museum 3, pl. 33, $1 \mathrm{~A}-\mathrm{C}$. Sur un chous attique à figures noires, il est face à un seul couple de joueurs; Munich, Antikensammlungen 6092 (milieu Ve s. av. J.-C.); G. vAN HOORN, Choes and Anthesteria, Leiden, 1951, nº 690, fig. 282 ; wÜNSCHE, KNAUSS, o.c. (n. 1), p. 428, fig. 47.6, cat. 251.

38. .Tubingen, Eberhard-Karls-Universität, Archäologisches Institut 5643; $\operatorname{ARV}^{2} 1127,8$; BA 214 889. Sur un loutrophore du même peintre, Éros apporte en volant deux fruits à la mariée ; New York, Metropolitan Museum of Art 1.73, $A R V^{2} 1126,6$; R.D. sutToN, « Nuptial Eros: The Visual Discourse of Marriage in Classical Athens ", JWAG 55-56 (1997/1998), p. 32-33, fig. 8 (interprétés comme les fruits qu'elle va consommer dans la chambre nuptiale). Sur la fonction de ces vases dans les rites de mariage, V. SABETAI, «Women's Ritual Roles in the Cycle of Life ", in N. KALTSAS, A. SHAPIRO (éd.), Worshipping Women, Ritual and Reality in Classical Athens, Athens, 2008, p. 289-297 ; EAD., "The Wedding Vases of the Athenians: a View from Sanctuaries and Houses ", in M.-Chr. VILLANUEVA (dir.), Des vases pour les Athéniens (VI ${ }^{e}-I V^{e}$ siècles avant notre ère), Métis 12 (2014), p. 51-79. 39. Théocrite, Idylles II. 
40. .Londres, British Museum GR 1874.5.12.1 ; LISSARRAGUE 1996, o.c. (n. 6), p. 423, fig. 98B. Voir aussi les deux paires de boucles d'oreilles de mariage avec Éros manipulant une iunx, tandis qu'au sommet Niké joue aux osselets (de Kyme? vers 330-300 av. J.-C.; Londres, British Museum GR 1877.9-10.16-17, 1877.9-10.18.-19 ; D. WILLIAMS, J. OGDEN, Greek Gold. Jewelry of the Classical World, London, 1994, p. 96-97, $\mathrm{n}^{\circ}$ 49-50.

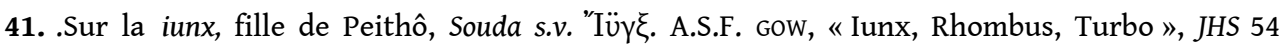
(1934), p.1-13 ; M. DETIENNE, Les jardins d'Adonis, Paris, 1972, p. 161-167 ; V. PIRENNE-DELFORGE, «L'Iynge dans le discours mythique et les procédures magiques », Kernos 6 (1993), p. 277-289 ; Chr. FARAONE, Ancient Greek Love Magic, Harvard, 1990.

42. .University of Chicago, the David and Alfred Smart Museum of Art 1967.115.347. PFISTERERHAAS, o.c. (n. 8), p. 175 , cat J 47, pl. 45,3.

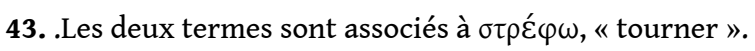

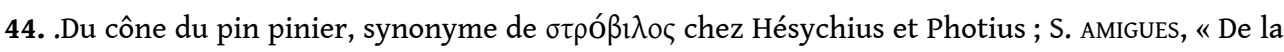

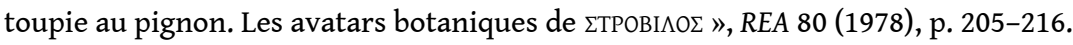

45. .Les deux mots suggèrent le vrombissement du mouvement rotatif ; F. SKODA, Le redoublement expressif : un universal linguistique, Paris, 1982, p. 68.

46. .Aristophane, Les oiseaux 1460-1469. Cf. Virgile, Enéide VII, 378-383 (trad. J. Perret, CUF) : "Avez-vous vu voler sous les coups de fouet la toupie que les enfants en grand cercle, attentifs à leur jeu, font tourner autour de l'atrium désert? Activée par la lanière, elle décrit des courbes rapides; la troupe enfantine, immobile, émerveillée, se penche sans comprendre, admire le buis qui tourne et qu'animent les coups. »

47. .Cf. le fameux poème de l'Anthologie Palatine VI, 309: "Philoclès a consacré à Hermès son ballon renommé, ses bruyantes castagnettes de buis, les dés qu'il a aimés à la folie et la toupie qu'il faisait tournoyer : tous les jouets de son enfance. » Voir aussi les toupies votives du Cabirion de Thèbes ; P. wolters, G. BRUNS, Das Kabirenheiligtum bei Theben, I, Berlin, 1940, p. 123-124, pl. 18, 2, 4-11 et pl. 19, 4-6, ainsi que la statuette votive en bronze étrusque de jeune homme avec une toupie, Munich, Antikensammlungen SL 25 ; VANHOVE, o.c. (n. 10), p. 164, cat. 18 ; WÜNSCHE, KNAUSS, o.c. (n. 1), p. 415, fig. 44.1 .

48. Baltimore, Johns Hopkins Archeological Museum B 9 ; ARV 2444,251 ; BA 205298 ; J. NEILS, J.H. OAKLEY (éd.), Coming of Age in Ancient Greece, Images of Childhood from the Classical Past, New Haven, 2003, p. 216, cat. 16. Coupe, Florence, Museo Archeologico etrusco 80528; ARV 2377,113; BA 20 411. Sur un lécythe à figures rouges, on relèvera la présence du sac d'astragale entre Hermès et le garçon; Tubingen, Archäologisches Institut S./10814; ARV 2 463,5; VANHOVE, o.c. (n. 10), p. 164, cat. 17.

49. .CALAME 1977, o.c. (n. 3), p. 414. Cf. Platon, Lois, 653 d-e sur la nature enfantine toujours en mouvement, incapable de rester au repos. Sur la dimension d'éducateur d'Hermès, D. JAILLARD, Configurations d'Hermès. Une 'théogonie hermaïque', Liège, 2007, p. 181-185.

50. .Sur la gestualité juvénile, T.J. MCNIVEN, «Behaving like a Child: Immature Gestures in Athenian Vase Painting ", in A. COHEN, J.B. RUTTER (éd.), Constructions of Childhood in Ancient Greece and Italy, Princeton, 2007, p. 85-99.

51. .Platon, République IV, 436d : «[...] les toupies sont toutes entières et dans le même temps en repos et en mouvement, quand, leur centre restant fixe, elles tournent sur elles-mêmes. » (trad. E. Chambry, CUF).

52. Dans l'Iliade XIV, 411-413, Ajax frappe Hector à la poitrine avec une pierre et l'envoie rouler

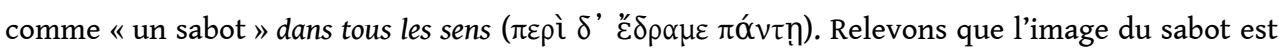
utilisée pour signifier métaphoriquement la discipline dans les Emblèmes de Jacob Cats ( $\left.\mathrm{xvII}^{\mathrm{e}} \mathrm{s}.\right)$; M.F. DURANTINI, The Child in Seventeenth-Century Dutch Painting, Ann Arbor, 1983, p. 114: "The top spins merrily on the floor, whipped by a biting cord, and the harder one hits the better it spins. But let up a bit with the whip and it falls in the dust. From then on it won't do a single turn, but lie forever like a block. 
One never watches it better than in times of sorrow and unhappiness. For if anyone lives without pain, he rusts at once from idleness. When man has too much leisure, you see, that's when the heart yearns for lust. " Je remercie Michel Manson de cette information, développée dans sa thèse de doctorat, Le jouet dans la France d'Ancien Régime, Université Paris 1, 1999.

53. .Le kônos et le rhombos se trouvent dans l'énumération du Papyrus de Gourob, mi-III ${ }^{\mathrm{e}}$ s. av. J.C., 1. 29, avec les astragales; F. GRAF, S.I. JOHNSTON, Ritual Texts for the Afterlife, Routledge, $2013^{2}$, p. 66-93, p. 152-153, p. 188-189. Voir aussi Clément d'Alexandrie, Exhortation aux Gentils 17 ; DETIENNE, o.c. (n. 22), p. 172 ; O. LEVANIOUK, « The Toys of Dionysos », HSCPh 103 (2007), p. 165-202.

54. .Tibulle, Elégies I, V, 3-5.

55. .Par ex. PGM XVIIa 1-25: « [...] jusqu'à ce que, fouettée ( $\mu \alpha \sigma \tau i \zeta \omega)$ par Anubis, elle vienne me désirant ardemment. » (trad. G. Ficheux, o.c. [n. 34], p. 134-135). Sur l'image du fouet, FARAONE, o.c. (n. 41), p. 60-64.

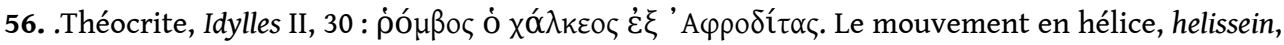
$\mathrm{du}$ fuseau dans les mains des femmes n'aurait-il pas aussi une valeur divinatoire et de magie amoureuse ? Les Moires filent le destin, et l'art d'entrelacer la trame est une métaphore de l'union sexuelle; F. frontisi-ducroux, Ouvrages de dames. Ariane, Hélène, Pénélope..., Paris, 2009, p. 47-50.

57. .Matera, Museo Nazionale Domenico Ridola ; G. SCHNEIDER-HERRMANN, A.D. TRENDALL, « Eros with a Whipping-Top on an Apulian Pelike ", BABesch 50 (1975), p. 267-282; K. SCHAUENBURG, «Erotenspiele, 1. Teil », Antike Welt 7 (1976), p. 45, fig. 24.

58. .Syracuse, Museo Arch. Regionale Paolo Orsi 36465 ; BA 9003794 ; CVA Syracuse, 1, IV E 6, pl. 10,3 ; SCHAUENBURG, o.c. (n. 57), p. 42, fig. 14.

59. Sur cette spécialité d'Hermès, voir Apollodore, Bibliothèque, III, 10, 2 ; Pausanias, VII, 22, 2-4. D. JAILLARD, « Hermès et la mantique grecque ", in S. GEORGOUDI, R. KOCH PIETTRE, F. SCHMIDT (dir.), La raison des signes Présages, rites, destin dans les sociétés de la Méditerranée ancienne, Leiden/ Boston, 2012, p. 91-107, spéc. p. 92-96. Cf. les oracles tirés avec des osselets, F. GRAF, « Rolling the Dice for an Answer ", in S.I. JOHNSTON, P.T. STRUск (éd.), Mantikê. Studies in Ancient Divination, Leiden, 2005, p. 51-97; J. NOLLÉ, Kleinasiatische Losorakel: Astragal- und Alphabetchresmologien der hochkaiserzeitlichen Orakelrenaissance, München, 2007.

60. .Anthologie Palatine VII, 89 (= Callimaque, Épigrammes I, 8).

61. .Trad. P. Waltz, CUF. Pour un autre exemple d'interprétation divinatoire de jeux d'enfants, cf. Plutarque, Isis et Osiris, 356 e. P. courcelle, «L'oracle d'Apis et l'oracle du jardin de Milan (Augustin, « Conf. », VIII, 11, 29) », RHR 139 (1951), p. 216-231, en déduit que les oracles d'Apis de Memphis, que Callimaque devait connaître, étaient livrés par ce type de jeux d'enfants dans le temple. Sur l'usage d'enfants pour tirer les sorts, S.I. JOHNSTON, «Charming Children: The Use of the Child in Ancient Divination », Arethusa 34 (2001), p. 97-111.

62. .New York, Metropolitan Museum of Art 1875, 75.2.9; BA 9515 ; NEILS, OAKLEY, o.c. (n. 48), p. 270 , cat. 77.

63. .Cette exigence explique le choix d'Achille et d'Ajax comme partenaires dans la fameuse scène de jeu; V. DASEN, « Achille et Ajax : quand l'agôn s'allie à l'alea », Revue du MAUSS 46 (2015), p. 8198.

64. .Athènes, Musée national, ex coll. Vlastos; ARV 245.163 ; BA 212311 ; J.H. OAKLEY, Picturing Death in Classical Athens. The Evidence of the White Lekythoi, Cambridge, 2004, p. 56, fig. 33.

65. .H. HOFFMANN, Sotades: Symbols of Immortality on Greek Vases, Oxford, 1997, p. 141, signale que le mot pourrait venir du grec $\mu$ ópoc, le destin, surtout fatal, associé à $\mu$ oĩ $\alpha$, la « part » attribuée par le destin. Sur le jeu moderne, voir par ex. P. COLletTA, La Mourra Bella: histoire et histoires d'un jeu interdit, Nice, 2006 (La Sardaigne, la Corse et l'Italie du sud).

66. .Une variante moderne autorise à lever deux mains, ou à lever le poing qui vaut zéro, mais aucun texte ni image n'en témoigne à ce jour. 
67. .K. SCHNEIDER, « Micare », RE XV 2 (1932), col. 1516-1517.

68. Sur la divination corporelle, fondée sur les tressaillements involontaires du corps, par ex. V. DASEN, J. WILGAUX, « De la palmomantique à l'éternuement, lectures divinatoires des mouvements du corps ", Kernos 26 (2013), p. 111-122.

69. Cicéron, De la divination II, 41, 85 (trad. G. Freyburger et J. Scheid, CUF) : Quid enim sors est? Idem propemodum, quod micare, quod talos jacere, quod tesseras; quibus in rebus temeritas et casus, non ratio nec consilium ualet. Tota res est inuenta fallaciis, aut ad quaestum, aut ad superstitionem, aut ad errorem.

70. .Calpurnius Siculus, Bucoliques II, 25-27 (trad. J. Amat, CUF). Voir aussi Nonnos, Dionysiaques XXXIII, 77 : Éros et Hyménée jouent à «bouge-doigts » pour savoir qui lancera le cottabe le premier.

71. .Cicéron rappelle dans Les devoirs II, 19, 77 combien la vigilance était importante : « quand ils louent la bonne foi de quelqu'un et sa bonté, ils disent qu'il est digne qu'on 'joue à la mourre avec lui dans l'obscurité', quicum in tenebris mices" (trad. M. Testard, CUF).

72. .Voir le catalogue établi par H.A. CAHN, « Morra: Drei Silene beim Knobeln », in H. FRONING, T. HÖLSCHER, H.F. MIELSCH (éd.), Kotinos, Festschrift für Erika Simon, Mainz, 1992, p. 214-217 ; V. DASEN, «Le destin au bout des doigts », in A. NEUMANN-HARTMANN, Th. S. schmidt (éd.), Munera Friburgensia. Festschrift zu Ehren von Margarethe Billerbeck, Bern, 2016, p. 167-175.

73. Cratère en cloche apulien, Berlin, coll. Graf Hohenthal, vers 410 av. J.-C.; SCHAUENBURG, o.c. (n. 57), p. 40, fig. 9 ; CAHN, o.c. (n. 72 ), $\mathrm{n}^{\circ} 5$.

74. .Sur le gestus computationis ou comput digital, bien documenté pour le monde romain (Quintilien, Art oratoire VII, 10, 35), G. MINAUD, « Des doigts pour le dire. Le comput digital et ses symboles dans l'iconographie romaine », Histoire et Mesure (2006), p. 3-34 ; J. GAVIN, A. SCHÄRLIG, Sur les doigts, jusqu'à 9999. La numération digitale, des Anciens à la Renaissance, Lausanne, 2014.

75. .Munich, Antikensammlung 3268 ; SCHAUENBURG, o.c. (n. 57), p. 40-41; CAHN, o.c. (n. 72), $\mathrm{n}^{\circ} 4$; WÜNSCHE, KNAUSS, o.c. (n. 1), p. 376-377, fig. 36.8-9, p. 419, fig. 45.1, cat. 103.

76. .Cf. les inscriptions dans quelques scènes du jeu d'Achille et Ajax, DASEN, o.c. (n. 63), p. 84 (les chiffres 2, 3, et 4).

77. Bâle, Coll. Cahn HC 431 ; BA 44585 (P. de Sotades). CAHN, o.c. (n. 72), nº 1, pl. 45, 2-3 ; HOFFMANN o.c. (n. 62), p. 141-143, fig. 79 ; F. LISSARRAGUE, La cité des satyres. Une anthropologie ludique (Athènes, $\mathrm{VI}^{e}-V^{e}$ siècle avant J.-C.), Paris, 2013, p. 208-209, fig. 179 ; V. DASEN, « Jeux de mains, jeux de vilains? La morra ", Archéothema. Jeux et jouets gréco-romains 31 (2013), p. 37.

78. Sur le jeu des satyres, LISSARRAGUE 2013, o.c. (n. 77), p. 241, fig. 209 (ephedrismos avec Éros).

79. .LISSARRAGUE 2013, o.c. (n. 77), p. 63-66.

80. .Naples, Musée archéologique 82038, H 2574 ; SCHAUENBURG, o.c. (n. 57), p. 41, fig. 10 ; CAHN, o.c. (n. 72), $n^{\circ} 9$; WÜNSCHE, KNAUSS, o.c. (n. 1), p. 420, fig. 45.3. 1. Derrière la jeune fille, une femme tient aussi une couronne.

81. Cf. la jeune fille assise sur un coffre sur une coupe apulienne de Bari; sCHAUENBURG, o.c. (n. 57), p. 41, fig. 1 ; CAHN, o.c. (n. 72), $\mathrm{n}^{\circ} 8$.

82. Goluchow, Musée archéologique national 14299.3; $A R V^{2} 1130,150$; BA 214960 ; O. JAHN, «Giocatrici a morra », Annali dell'Istiluto di Corrispondenza archeologica 38 (1866) 326-329; CAHN, o.c. (n. 72), $\mathrm{n}^{\circ} 2$.

83. Sur les lustrations prénuptiales, voir n. 6. Sur l'érotisme de l'espace des fontaines, V. SABETAI, "The Poetics of Maidenhood: Visual Constructs of Womanhood in Vase-Painting ", in S. SCHMIDT, J.H. OAKLEY (éd.), Hermeneutik der Bilder. Beiträge zur Ikonographie und Interpretation griechischer Vasenmalerei, Munich, 2009, p. 103-114, spéc. p. 107, fig. 4.

84. .Athènes, Musée National $332 ; A R V^{2}$ 1257,2 ; BA 217056. H. METZGER, « Ôon à figures rouges ", Monuments et mémoires de la Fondation Eugène Piot 40 (1944), p.69-86 (= in P. AMANDRY [dir.], 
Collection Hélène Stathatos, III, Objets antiques et byzantins, Strasbourg, 1963, p.160-179). Il proviendrait d'une tombe dont le mobilier a été dispersé ; CAHN, o.c. (n. 72), $\mathrm{n}^{\circ}{ }_{3}$; WÜNSCHE, KNAUSS, o.c. (n. 1), p. 420, fig. 45.2 .

85. .Dans d'autres interprétations, la jeune femme assise serait la mère consultant Aphrodite au sujet de l'avenir de sa fille; sur les différentes hypothèses depuis 1944, voir METZGER, o.c. (n. 84), p. 172-174.

86. .METZGER, o.c. (n. 84), p. 174-179, fig. 80-81.

87. Boston, Museum of Fine Arts 99.533 ; K. BRAUN, Th. E. HAEVERNICK, Das Kabirenheiligtum bei Theben, IV, Berlin, 1981, p. 65, $\mathrm{n}^{\circ} 366$; CAHN, o.c. (n. 72), $\mathrm{n}^{\circ} 6$; A.G. MITCHELL, Greek Vase-Painting and the Origins of Visual Humour, Cambridge, 2009, p. 270, fig. 140 (la femme tenant la couronne serait Aphrodite) ; V. DASEN, o.c. (n. 75), p. 37 ; GHERCHANOC, o.c. (n. 17), p. 40-41, fig. 11.

88. .Sur les inventions mythographiques de Ptolémée Hephestion ou Chennos, A. CAMERon, Greek Mythography in the Roman World, Oxford, 2004, p. 134-159.

89. .Photius, Bibliothèque 149a, 16-18 (trad. R. Henry, CUF) ; F. DORIA, Severe ludere. Uso e funzione dell'astragalo nelle pratiche ludiche e divinatorie del mondo greco, Cagliari, 2012, p. 48.

90. .Photius, Bibliothèque, 149b, 8-12 (trad. R. Henry, CUF). Sur la dispute des parents sur le nom de l'enfant, où la mère a autant de poids que le père, cf. Aristophane, Nuées, 59-67.

91. .Anacréon, fr. 398. Cf. Méléagre, Anthologie Palatine XII, 47 (trad. R. Aubreton, F. Buffière, CUF) : «Encore aux bras de sa mère, jouant de bon matin aux osselets, Éros, ce bambin, a joué ma vie.».

92. .Voir aussi J.R. GREEN, "Zeus on a See-Saw. A Comic Scene from Paestum », Logeion. A Journal of Ancient Theatre 4 (2014), p. 1-27. Je remercie Martine Denoyelle de m'avoir signalé cet article.

93. Sur le jeu qui donne de la chance, R. HAMAYON, Jouer. Une étude anthropologique à partir d'exemples sibériens, Paris, 2012, p. 226-252, spéc. p. 231-232.

\section{RÉSUMÉS}

Sur les vases attiques et italiotes, plusieurs jeux d'adresse et de hasard mettent en scène des jeunes gens des deux sexes en âge de se marier. L'objectif des imagiers n'est pas de représenter un jeu réel, permettant de reconstituer des règles. Les pratiques ludiques transposent dans un univers virtuel le prélude des relations amoureuses et les préparatifs du mariage. Les jeux d'adresse et de hasard créent un espace métaphorique où les jeunes filles ne sont pas vues comme de simples objets du désir masculin. La dynamique du jeu visualise la représentation collective et sociale de leur monde intérieur placé sous le signe de l'incertitude, de l'émotion; leur activité ludique s'apparente aux pratiques divinatoires et magiques, patronnées par Éros et Aphrodite.

On Attic and Italiot ceramics, several skill and chance games involve young individual of both sexes in prenuptial age. The aim of the painters is not to represent a real game, allowing the reconstruction of rules. Ludic activities transpose courtship and the preparations of wedding in a virtual world. Games of skill and chance create a metaphorical space where girls are not seen as objects of male lust. The dynamic of games visualises the collective and social representation of their interior world made of uncertainty and emotion; their ludic activities are akin to oracular and magical practices, under the patronage of Eros and Aphrodite. 
AUTEUR

VÉRONIQUE DASEN

Université de Fribourg

Institut du Monde Antique et Byzantin

16 , rue Pierre-Aeby

$\mathrm{CH}-1700$ Fribourg

veronique.dasen@unifr.ch 